\title{
High-Accuracy Mass Determination of Unstable Cesium and Barium Isotopes
}

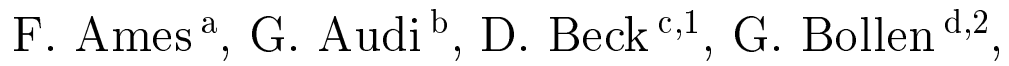 \\ M. de Saint Simon ${ }^{b}$, R. Jertz ${ }^{\mathrm{a}, 3}$, H.-J. Kluge ${ }^{\mathrm{c}}$, A. Kohl ${ }^{\mathrm{c}}$, \\ M. König ${ }^{c, 4}$, D. Lunney $^{\mathrm{b}}$, I. Martel ${ }^{\mathrm{e}}$, R.B. Moore ${ }^{\mathrm{f}}$, T. Otto ${ }^{\mathrm{a}, \mathrm{d}}$, \\ Z. Patyk ${ }^{g}$, H. Raimbault-Hartmann ${ }^{\mathrm{d}, 5}$, G. Rouleau ${ }^{\mathrm{f}, \mathrm{d}}$, \\ G. Savard ${ }^{\mathrm{a}, 6}$, E. Schark ${ }^{\mathrm{a}, 7}$, S. Schwarz ${ }^{\text {c }}$, L. Schweikhard ${ }^{\mathrm{a}}$, \\ H. Stolzenberg ${ }^{\mathrm{a}, 8}$, J. Szerypo ${ }^{\mathrm{h}, 9}$, and \\ the ISOLDE Collaboration ${ }^{\mathrm{d}}$ \\ ${ }^{a}$ Institut für Physik, Johannes Gutenberg-Universität, D-55099 Mainz, Germany \\ ${ }^{\mathrm{b}}$ CSNSM-IN2P3-CNRS, F-91405 Orsay-Campus, France \\ ${ }^{\mathrm{c}}$ GSI, Postfach 110552, D-64220 Darmstadt, Germany \\ ${ }^{\mathrm{d}}$ CERN, CH-1211 Geneva 23, Switzerland \\ ${ }^{\mathrm{e}}$ Instituto de Estructura de la Materia, CSIC, Madrid, Spain \\ ${ }^{\mathrm{f}}$ Foster Radiation Laboratory, McGill University, Montreal, H3A 2B1, Canada

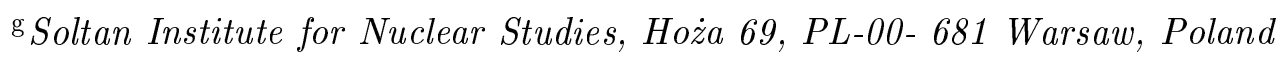 \\ ${ }^{\mathrm{h}}$ Institute of Experimental Physics, Warsaw University, PL-00-681 Warsaw, \\ Poland
}

PACS number: 21.10.Dr, 27.60.+j, 32.10.Bi, 07.75.+h

\begin{abstract}
Direct mass measurements of short-lived Cs and Ba isotopes have been performed with the tandem Penning trap mass spectrometer ISOLTRAP installed at the online isotope separator ISOLDE at CERN. Typically, a mass resolving power of 600000 and an accuracy of $\delta \mathrm{m} \approx 13 \mathrm{keV}$ have been obtained. The masses of ${ }^{123,124,126} \mathrm{Ba}$ and ${ }^{122 m} \mathrm{Cs}$ were measured for the first time. A least-squares adjustment has been performed and the experimental masses are compared with theoretical ones, particularly in the frame of a macroscopic-microscopic model.
\end{abstract}

Key words: ATOMIC MASSES ${ }^{117-142} \mathrm{Cs},{ }^{123-128,131,138-144} \mathrm{Ba}$; Measured masses. On-line mass spectrometry. Penning trap. Least-squares adjustment of data. Macroscopic-microscopic mass model calculations. 


\section{Introduction}

The binding energy of the atomic nucleus is one of the most fundamental properties of such a many-body system. Accurate mass data serve as testing grounds for nuclear models and stimulate their further improvement. Furthermore, systematic investigation of the binding energy as a function of proton and neutron number allows the direct observation of nuclear properties like pairing, shell and subshell closures, as well as deformation effects, and leads to a deeper understanding of nuclear structure. Large efforts are presently devoted, at several nuclear physics laboratories around the world, to apply new mass spectrometric techniques, such as time-of-flight, Smith-RF or Schottky mass spectrometry, for the accurate mass determination of short-lived isotopes far from the valley of beta stability [1].

A particularly successful approach has proven to be the use of a Penning trap as a mass spectrometer. Here we report on results obtained by ISOLTRAP which is a Penning trap mass spectrometer installed at the on-line mass separator facility ISOLDE at CERN in Geneva. During its first decade of operation ISOLTRAP has been steadily improved and presently offers an accuracy in mass determinations of $10^{-7}$ or better. It is applicable to all ion beams available at ISOLDE of isotopes with half-lives down to about one hundred milliseconds.

Highly accurate mass measurements far from stability have to date been carried out on isotopic chains of alkali and alkali earth isotopes [2,3], on rare earth isotopes [4] and most recently on neutron deficient mercury isotopes and isotopes of various elements in the vicinity of ${ }^{208} \mathrm{~Pb}[5,6]$.

In this paper we report on mass measurements on radioactive cesium and barium isotopes for ${ }^{117-142} \mathrm{Cs}$ and ${ }^{123-128,131,138-144} \mathrm{Ba}$. Typically, the masses were determined with a resolving power of $R \approx 600000$ and an accuracy of

\footnotetext{
1 present address: Instituut voor Kern- en Stralingsfysica, Celestijnenlaan 200 D, B-3001 Leuven, Belgium

2 corresponding author: georg.bollen@cern.ch, fax/phone: +41 22767 8990/5825

3 present address: Bruker Franzen Analytik GmbH, Fahrenheitstr. 4, D-28359 Bremen, Germany

4 present address: Data Care AG, Eisenstrae 58, D-65428 Rsselsheim

5 present address: Dresdner Bank AG, D-60301 Frankfurt, Germany

6 Argonne National Laboratory, 9700 S. Cass Avenue, Argonne, IL 60439, USA

7 present address: Arthur Andersen, Mergenthalerallee 10-12, D-65760 Eschborn

8 present address: Bauboden Systemhaus GmbH, Münchfeldstr.1-5, D-55122 Mainz, Germany

${ }^{9}$ present address: Laboratoire de Physique Corpuskulaire de Caen, F-14050 Caen Cédex, France
} 
$\delta m \approx 13 \mathrm{keV}$. The masses of ${ }^{122} \mathrm{Cs}^{m}$ and ${ }^{123,124,126} \mathrm{Ba}$ have been measured for the first time. The measurements have been carried out in several beam times spread over a period of several years. In the present paper the complete set of results will be presented and discussed, including the results of the very first series of measurements already published [2] and the data on ${ }^{123,125,127,131} \mathrm{Ba}$ published recently [4]. Except for the latter barium isotopes all data were included in the latest update of the mass tables of 1995 [7]. The adjustment procedure will be discussed as well as the large impact of the ISOLTRAP measurements on the general evaluation of atomic masses. The mass data will be compared with the results of nuclear mass models. Work will be presented in which the ISOLTRAP data have been used for a local adjustment of a macroscopic-microscopic mass formula.

\section{The Principle of Penning Trap Mass Determination}

The ion storage method has become a widely applied technique. The theory of the Penning trap and its applications are described in detail in a number of publications [8-13]. Therefore only a brief description will be given here, concentrating on the principle of Penning trap mass spectrometry as applied in the case of ISOLTRAP.

Charged particles are stored in a Penning trap by a superposition of a homogeneous magnetic field and an axial electrostatic quadrupole field. The motion of an ion confined in such an ideal trap can be described as a superposition of three independent harmonic oscillations: an axial oscillation with frequency $\nu_{z}$ along the magnetic field lines and two circular motions perpendicular to the magnetic field with frequencies $\nu_{+}$(reduced cyclotron motion) and $\nu_{-}$ (magnetron motion). All frequencies depend on the trapping voltage but in a uniform magnetic field and a pure quadrupolar electrostatic field the sum of the two radial frequencies equals the cyclotron frequency $\nu_{c}$ given by

$$
\nu_{+}+\nu_{-}=\nu_{c}=\frac{q}{2 \pi m} \cdot B
$$

It has been shown that by using a radio-frequency ( $\mathrm{RF}$ ) azimuthal quadrupole it is possible to excite the ion motion directly at the sum frequency $\nu_{+}+$ $\nu_{-}[12,13]$. If the ions are initially prepared in a pure magnetron orbit the application of such an RF field at this frequency $\nu_{c}$ with well chosen amplitude and interaction time $T_{R F}$ will convert the ion motion in the radial plane into a pure cyclotron motion. This is accompanied by a large increase in the kinetic energy in the radial plane, which is used to detect the resonance by a time-offlight technique [14]: The ions are ejected from the trap after excitation and are allowed to drift through the fringe field of the magnet to a channel plate 
detector where the time of flight is measured. During their adiabatic passage through the inhomogeneous part of the magnetic field the radial energy gained by the RF excitation is converted into axial energy. This leads in resonance to a reduction in the time of flight from the trap to the detector.

The resolving power of this experiment is determined by the interaction time $T_{R F}$ with the RF field. In the measurements reported here the interaction time was generally chosen to be $T_{R F}=0.9 \mathrm{~s}$. This corresponds to a Fourier-limit of about $1.2 \mathrm{~Hz}$ for the half-width of the cyclotron resonances and a resolving power of $R=\nu_{c} / \Delta \nu_{c}(F W H M) \approx 600000$ for nuclides in the mass region of interest here. In off-line measurements a resolving power of $R \approx 8000000$ was obtained for stable Cs by using a longer interaction time $\left(T_{R F}=12 \mathrm{~s}\right)$ and a correspondingly lower amplitude for the $\mathrm{RF}$ excitation. The resolving powers given here correspond to mass resolutions of $\delta m(F W H M) \approx 200 \mathrm{keV}$ and $\delta m(F W H M) \approx 13 \mathrm{keV}$, respectively.

\section{Experimental Setup and Procedures}

The tandem Penning trap mass spectrometer ISOLTRAP was first installed at the on-line mass separator ISOLDE-II at CERN. After the move of ISOLTRAP to the new PSB-ISOLDE facility [15], a number of mass measurements in the cesium and barium isotopic chains have been repeated and new determinations have been performed.

Figure 1 is a schematic of the setup as it was used for most of the measurements reported here. It consisted of two main parts, each adapted to a well defined task. The first part was a Penning trap in an electromagnet. Its purpose was to prepare the radioactive ions for the mass measurement. The second part, a high-precision Penning trap in a superconducting magnet, was used for the actual mass measurement. The drift tubes above the second Penning trap and the ion detector were used for detection of the cyclotron resonance. A detailed description of the apparatus can be found in [16]. A recent modification is presented in [17].

The mass measurement started with the collection of the radioactive ions delivered by the ISOLDE on-line mass separator. The ions were implanted into a rhenium foil placed below the first Penning trap. Then the foil was turned and heated by a pulsed current. In this way the radioactive atoms were released and surface-ionized. The resulting ions were trapped and cooled by collisions with a buffer gas. Simultaneously the ion motion was centered in the trap by a quadrupole excitation $[13,18]$. This centering technique is mass selective and removes isotopic contaminations delivered by the mass separator. The ions were then ejected from the trap and transferred to the second trap 
by an ion optical system. Here the ion pulse was captured in flight [19]. The remaining isobaric contaminations were removed by exciting their cyclotron motion using a dipole electric field at their reduced cyclotron frequency $\nu_{+}$. Subsequently the ion motion was excited by the azimuthal quadrupole RF field used for the mass measurement. For the detection of the cyclotron resonance the ions were ejected into the drift section and their time of flight from the trap to the detector was observed. Some 20 ions were detected in each cycle and the mean value of their time of flight was determined.

This cycle was repeated with different excitation frequencies, scanning the expected resonance frequency. The determination of the mean time of flight as a function of the applied frequency yields a resonance curve as shown in Fig. 2 , in which several scans have been added to obtain sufficient statistics. This example shows the cyclotron resonance of ${ }^{118} \mathrm{Cs}$. The measurement took $25 \mathrm{~min}$ and corresponds to a total of 4300 detected ions. The width of the resonance is $\Delta \nu_{c}(F W H M) \approx 1.2 \mathrm{~Hz}$ corresponding to $\Delta m(F W H M) \approx 180 \mathrm{keV}$ and to a resolving power of $R \approx 640000$.

A Gaussian was fitted to the resonance data, as shown in Fig. 2. This yields the resonance frequency and its statistical error, typically of the order of $\delta \nu_{c}=0.03 \mathrm{~Hz}$ for $10^{4}$ detected ions corresponding to $\delta \nu_{c} / \nu_{c} \approx 5 \cdot 10^{-8}$. A fit by the true theoretical line shape having small side bands [13] does not change significantly the value of the center frequency of the resonance but improves slightly the statistical accuracy. In view of the larger estimated systematic error, of a maximum of $1 \cdot 10^{-7}$ (see below), a fit by a Gaussian is regarded as sufficient.

The magnetic field has to be known in order to use eq. (1) to convert the measured frequencies into mass values. This was determined by frequently measuring the cyclotron frequency of a reference nuclide with a well known mass before, during and after an on-line run. In this way the uncertainty due to a possible drift of the magnetic field of the superconducting magnet was kept well below $1 \cdot 10^{-7}$.

Finally, for each nuclide under investigation the mean value $\bar{r}$ of the ratios of the cyclotron frequencies $r=\nu_{\text {ref }} / \nu$ of all runs, and its error, was calculated. To account for the sum of systematic errors due to an incomplete correction of magnetic field drifts and possible mass dependent effects that arise in an online experiment, an estimated systematic error of $1 \cdot 10^{-7}$ is added quadratically to the error of the averaged frequency ratio [16].

The frequency ratio $\bar{r}$ and its error is the final direct result of an ISOLTRAP mass comparison. It can be converted into an atomic mass value $m$ by multiplying the frequency ratio with the atomic mass of the reference isotope $m_{r e f}$ 
and taking into account the electron mass $m_{e}$ by

$$
m=\bar{r} \cdot\left(m_{r e f}-m_{e}\right)+m_{e} .
$$

The contribution of the binding energy of the electron is small compared to the total error of the present measurements and therefore neglected.

\section{Measurements}

The mass measurements on unstable $\mathrm{Cs}$ and $\mathrm{Ba}$ isotopes reported in this paper were performed in seven different runs, first at the on-line mass separator ISOLDE-II and later at the PSB-ISOLDE. In runs \#6 and \#7, a modified ISOLTRAP setup has been used where the electromagnet housing the first Penning trap has been replaced by a superconducting magnet [17]. Different projectile/target combinations for production of radioactive nuclides were used as well as tantalum and tungsten for surface ionization of the reaction products. Table 1 summarizes these data together with the investigated nuclides. The isotope ${ }^{133} \mathrm{Cs}$ was chosen as reference mass for the calibration of the magnetic field. The reason for this is twofold: firstly, this nuclide has a mass number which lies near the middle of the investigated mass range. This minimizes possible calibration errors [12]. Secondly, an auxilliary ion source for this stable nuclide was available, so that measurements on the reference could be performed without using the ISOLDE mass separator. All in all, the masses of 41 cesium and barium isotopes were determined, most of them in several runs and under different conditions (Table 1).

The analysis of the data and a series of test measurements showed that, besides the magnetic field variations described above, the only frequency shifts with an amplitude larger than $10^{-7}$ are due to contaminations of the investigated nuclides by other nuclear species with different masses [20]. These shifts are due to the Coulomb interaction of two ion species having different massover-charge ratios $m / q$ stored simultaneously in the trap. It was found [20] that when the mass difference between the two components is comparable to the line width of the cyclotron resonance, the RF-field interacts with the center of gravity $(1 / N) \sum_{i=1}^{N}(m / q)_{i}$ and a single resonance is observed with the same line width as in the case of a one-component ion cloud. $N$ denotes the number of stored ions. In the case of larger mass differences two cyclotron resonances are observed which are always broadened and both shifted towards lower frequencies. The size of the shift is directly proportional to the number of contaminating ions.

In the experiments discussed here we attempted to remove all contaminations. Nuclides with mass numbers different from the one investigated are 
largely suppressed by the ISOLDE mass separator. The remaining impurities are removed in the first Penning trap by the mass selective cooling process [18]. Therefore only isobars and isomers have to be considered as possible contaminants.

\subsection{Contamination by Isobars}

Since the ions are surface ionized in the ion source of the ISOLDE mass separator as well as on the foil in the first trap, only isobaric contamination by barium isotopes have to be considered in the cesium measurements and vice versa for the barium measurements.

For the cesium measurements the lower ionization potential of this element, as well as its shorter diffusion time out of the collection foil, suppressed the isobaric barium contaminations. Therefore the ISOLDE ionizer in the mass separator and the collection foil were operated at low temperatures.

For the measurements of neutron-rich barium isotopes the shorter half-life of the cesium isobar allowed its presence to be reduced by introducing a suitable delay between collection and the start of the measurement. In addition, a cleaning procedure was performed in the second trap to remove any remaining contamination. By using a dipole field at $\nu_{+}$to excite their cyclotron motion any remaining isobaric impurities were radially ejected from the trap before the excitation of the investigated isotope was started. This cleaning procedure was especially important for the neutron-deficient barium isotopes which decay into their cesium isobars during the collection in the ionizer foil.

\subsection{Contamination by Isomers}

Whereas none of the investigated barium isotopes have isomers with half-lives longer than one second, twelve of the cesium isotopes investigated have longlived isomers. In the studies reported here the cleaning process mentioned above was not applicable for isomers because of the small mass differences between the ground and the isomeric states.

In the case of cesium isotopes with mass number $A=123,124,130,134,135$, 136 and 138 the large difference in half-lives between ground and isomeric states was employed to avoid contamination. After the collection of the activity on the foil the start of the measurement was delayed by several half-lives of the shorter-lived state to let it decay.

In the case of ${ }^{122} \mathrm{Cs}$ it was possible to determine the excitation energy of the 
isomeric states. The isotope ${ }^{122} \mathrm{Cs}$ has two nuclear states with measured halflives of $21 \mathrm{~s}$ and $3.7 \mathrm{~min}$. Before our work it was assumed [21] that the shortlived state was the ground state and the excitation energy of the isomer was unknown. The cyclotron resonance of the pure 3.7 min activity was measured in this experiment after a delay of $2 \mathrm{~min}$ to let the $21 \mathrm{~s}$ state decay. Other measurements were started immediately after collection, thus having nuclei in both states in the trap.

The measured resonance lines are shown in Fig. 3, in the upper part for the pure $T_{1 / 2}=3.7 \mathrm{~min}$ state and in the lower part for the mixture. In the latter case, the contamination led to a positive frequency shift of about $0.5 \mathrm{~Hz}$, corresponding to a lower mass. It is interesting to note that the two resonances have the same width and the same time-of-flight reduction amplitude. This indicates that all ions in the trap contribute to the resonance and the center of gravity of the stored ion cloud is excited.

Since the $\left(T_{1 / 2}=3.7 \mathrm{~min}\right)$-state has the low cyclotron frequency it can be concluded that the $\left(T_{1 / 2}=21 \mathrm{~s}\right)$-activity is the ground state. To obtain the excitation energy of the isomer, the cyclotron resonances were measured with different delays between collection and start of the measurement. The result is given in Fig. 4, where the shift of the resonance frequency relative to the cyclotron frequency of the pure $3.7 \mathrm{~min}$ isomer is plotted versus the delay time. The dashed line is the result of a fit to the data under the assumption that it is the resonance of the center of gravity that is detected. The free parameters of the fit, i.e. the excitation energy $E^{*}$ of the isomer and the relative intensity ratio $R_{m g}$ of the isomer to the ground state at time zero, are found to be $E^{*}=$ $135(14) \mathrm{keV}$ and $R_{m g}=0.9(0.2)$.

For the cesium isotopes with $A=117$ - 121 the half-lives of the ground state and of the excited isomer are nearly equal. Therefore, it was not possible to apply the procedure described above. As in the case of ${ }^{122} \mathrm{Cs}$ the width of the measured cyclotron resonances and the time-of-flight effect show no sign of contamination. It was concluded that in these cases the excitation energy of the isomer is smaller than the energy corresponding to the resonance line width and that the center of gravity was detected. Therefore, a correction can be applied to derive the ground state mass. We follow the procedure discussed in $[22]$ where the mass of the ground state

$$
m=m^{*}-\frac{R_{m g}}{R_{m g}+1} E^{*}
$$

is calculated from the measured mass $m^{*}$, the production ratio $R_{m g}$ of isomer to ground state and the estimated excitation energy $E^{*}$. The mass corrections $\Delta m=m^{*}-m$ to be applied to ${ }^{117-121}$ Cs isotopes are given in Table 2 . The values of $R_{m g}$ are those adopted in [22]. The values for $E^{*}$ in ${ }^{117-120} \mathrm{Cs}$ are 
derived from recent systematics of isomeric excitation energies [23]. It can be noted that these energies (estimated independently from the present work) as well as the known isomeric excitation energy $E^{*}=68.5(3) \mathrm{keV}$ in ${ }^{121} \mathrm{Cs}$ are smaller than the width of the cyclotron resonance of about $\Delta m(\mathrm{FWHM}) \approx$ $180 \mathrm{keV}$. This is consistent with the observation of a single narrow resonance which does not show a line broadening and a reduction of the time-of-flight effect as it would be expected for larger mass differences.

\section{Cyclotron Frequency Ratios}

Table 3 compiles the ratios of the cyclotron frequencies of the investigated and the reference isotopes as obtained in run \#1 - \#4 with the ISOLTRAP mass spectrometer installed at the ISOLDE-II on-line isotope separator. The two uncertainty values given are the statistical error and the total error which includes the estimated maximum systematic error of $1 \cdot 10^{-7}$. Marked by an asterix are isotopes for which a cyclotron frequency ratio was measured in more than one run and where column 2 gives the weighted average of these ratios. In all cases excellent consistency was observed between the results from the different runs within the limits given by the systematic error.

Table 4 lists the results of 14 measurements performed at the new PS-Booster location (run \#5-\#7) after complete dismounting and reassembly of the ISOLTRAP spectrometer. Since the most important contributions to the estimated systematic error of $1 \cdot 10^{-7}$ of run \#1 - \#4 and of run \#5 and \#6 are uncorrelated, the overlapping data for six isotopes in Table 3 and 4 are treated as independent measurements. A very good agreement between the mass values determined at ISOLDE-2 and the PS-Booster ISOLDE is observed except for ${ }^{140} \mathrm{Cs}$ (observed shift of frequency ratio: $\left.+1.5 \cdot 10^{-7}\right)$ and ${ }^{120} \mathrm{Cs}\left(+2.0 \cdot 10^{-7}\right)$.

As can be seen from the uncertainties listed in Tables 3 and 4, the quality of the cyclotron frequency determination is independent of the mass difference between the isotope under investigation and the reference isotope. This was checked for extreme cases of large mass difference at ISOLDE-II [5] and at the PS-Booster. In run \# 5 the cyclotron frequency of ${ }^{133} \mathrm{Cs}^{+}$was measured relative to that of ${ }^{226} \mathrm{Ra}^{+}$. The experimentally determined ratio (also given in Table 4) is $\nu_{c}\left({ }^{133} \mathrm{Cs}^{+}\right) / \nu_{c}\left({ }^{226} \mathrm{Ra}^{+}\right)=1.700651167(18)(171)$, where the first error indicates the statistical uncertainty and the second includes also the systematic one. The experimental ratio agrees within the error bars $(+0.8 \sigma)$ with the accepted values [7]. 


\section{Mass Values}

The experimental frequency ratios as given in Table 3 and 4 are the final direct result of an ISOLTRAP mass comparison. From these ratios mass values are readily derived by use of eq. (2) together with the adopted mass value for the reference isotope $M E\left({ }^{133} \mathrm{Cs}=88075.7(3.0) \mathrm{keV}\right.$ [7]. In column 3 of Table 5 the results are given for all cesium and barium isotopes investigated by ISOLTRAP. In the cases of ${ }^{117-121}$ Cs isomeric corrections have been applied according to Table 2. For comparison the results of the 1986 mass evaluation [24], for which Penning trap data had not yet been available, are given in column 4. Since then ISOLTRAP data have been used for several mass evaluations [25,7] which will be discussed in detail below. The result of the most recent mass update of 1995 [7] is given in column 5 of Table 5.

It is interesting to compare the directly obtained Penning trap mass values with the mass predictions of the 1986 evaluation [24]. Figures 5 and 6 show in the upper parts the difference between the 1986 mass values (zero line with error band) and the Penning trap data (points with error bars) for the cesium and barium isotopic chain. A systematic deviation is observed for neutrondeficient Cs and, less pronounced, for neutron-rich Ba isotopes. Strong disagreement shows up for ${ }^{130,135} \mathrm{Cs}$ and ${ }^{128} \mathrm{Ba}$.

\subsection{Mass adjustments with the ISOLTRAP Data}

A first preliminary global adjustment was performed including only the Penning trap data of run \#1 published in a letter [2]. In the 1993 mass evaluation [25] the data of the first four runs (run \#1 - \#4) were included. The ISOLTRAP data were used completely for the 1995 mass update [7], with exception of the very recently published data on ${ }^{123,125,127,131} \mathrm{Ba}$ [4].

For the mass adjustments the frequency ratios have to be converted into linear relations between the masses of the nuclides as explained and given in detail in Appendix A. The linear equations obtained are then entered into a general adjustment calculation (least-square adjustment) that takes account of all experimental information on nuclear masses available [25]. In this way can all the information contained in the measurements be extracted, the consistency with other data be checked and the impact on the nuclear mass landscape be evaluated.

In the first preliminary mass adjustment significant discrepancies were observed for ${ }^{130,135} \mathrm{Cs}$ and ${ }^{128} \mathrm{Ba}$ already mentioned above, and also for ${ }^{141} \mathrm{Ba}$, and ${ }^{142} \mathrm{Ba}$. Except for ${ }^{142} \mathrm{Ba}$ all discrepancies could be resolved as will be discussed below. 
The mass of the isotope ${ }^{135} \mathrm{Cs}$ was determined prior to the trap measurements by the $\mathrm{Q}$-values of the $\beta$-decays of ${ }^{135} \mathrm{Xe}$ and ${ }^{135} \mathrm{Cs}$. The $\mathrm{Q}$-value for ${ }^{135} \mathrm{Cs}$ was given in a conference abstract without any quoted error [26] and not published thereafter. The ISOLTRAP value agrees with the $Q_{\beta}$-value of ${ }^{135} \mathrm{Xe}$. In the meantime a $Q$-value measurement of the reaction ${ }^{134} \mathrm{Cs}(\mathrm{n}, \gamma){ }^{135} \mathrm{Cs}$ has been reported [27]. This measurement has the largest influence on the mass value of ${ }^{135} \mathrm{Cs}$ in the 1993 adjustment, is in very good agreement with the Penning trap value and is in strong disagreement with the value derived from [26]. The latter data was therefore excluded from the 1993 atomic mass evaluation and the 1995 update.

In the case of ${ }^{130} \mathrm{Cs}$ our value is determined from two different runs that agree reasonably well (Table 3 ) but is in strong conflict with the $\mathrm{Q}_{\beta}$-decay of ${ }^{130} \mathrm{Cs}$ from ref. [28]. If our value for ${ }^{130} \mathrm{Cs}$ is used, several (not severe) inconsistencies in this region vanish. The $\mathrm{Q}$-value of [28] was in conflict with all measurements determining the mass value of ${ }^{130} \mathrm{Cs}$, even with the $\mathrm{Q}_{\beta+^{-}}$ value of ${ }^{130} \mathrm{Cs}$ determined in the same publication. The reason for the strong disagreement was the estimated error of the $\mathrm{Q}_{\beta-}$-value [28] of only $4 \mathrm{keV}$. This error was assumed since no errors were given in the publication. However, in [28] not a pure $\beta^{-}$-spectrum was recorded but the sum of the $\beta^{+}$- and $\beta^{-}$-decays. The $\mathrm{Q}$-value for the $\beta^{-}$-branch was obtained from the difference between the sum spectrum and the highly energetic $\beta^{+}$-component. Since this procedure leads to substantial uncertainties an error for the Q-value of ${ }^{130} \mathrm{Cs}\left(\beta^{-}\right)$of $50 \mathrm{keV}$ was assumed for the further adjustments. This removed all discrepancies for this isotope.

For ${ }^{128} \mathrm{Ba}$ the discrepancy was due to the work of Debenham et al. [29]. There the mass difference between ${ }^{128} \mathrm{Ba}$ and ${ }^{130} \mathrm{Ba}$ was determined by a $(\mathrm{p}, \mathrm{t})$ reaction. Since they could not resolve the ground and excited state of ${ }^{128} \mathrm{Ba}$ in the measured tritium spectrum, the error of this measurement was increased by adding their estimated correction quadratically to the quoted error before performing the adjustments in the 1995 mass update.

For ${ }^{141} \mathrm{Ba}$ and ${ }^{142} \mathrm{Ba}$ there was a conflict between different input values. In the case of ${ }^{141} \mathrm{Ba}$ the Penning trap value was in agreement with two $\mathrm{Q}_{\beta}$-value measurements on ${ }^{141} \mathrm{Ba}$ and two on ${ }^{141} \mathrm{Cs}$. The conflict was due to a third measurement on ${ }^{141} \mathrm{Cs}$ [30]. In this work only the endpoint energies of the measured spectra were given. These values were erroneously used as Q-values in the older evaluation of masses [24]. Since ${ }^{141} \mathrm{Cs}$ does not decay into the ground state of ${ }^{141} \mathrm{Ba}$, the excitation energy $(48.5 \mathrm{keV})$ of the level to which it decays has to be added to the endpoint energy to obtain the Q-value. A recent remeasurement of this decay energy by the same group [31] confirms 
this result.

In the case of ${ }^{142} \mathrm{Ba}$ its mass is now determined by six measurements. The two Penning trap data agree with three of the other measurements and are in conflict only with [32] where the Q-value of ${ }^{142} \mathrm{Ba}$ was determined with high precision. No obvious error could be found in this work and its result was still kept as an input datum for the mass adjustments.

With the ISOLTRAP measurements a series of accurate data are now available. Within the mass adjustment this allowed to check earlier direct mass measurements performed with magnetic spectrometers in this mass region. It appeared from a careful analysis of the Orsay "triplet"-mass spectrometric data [33] and those of the St. Petersburg on-line prism mass spectrometer [34] that the actual uncertainties of both measurement procedures are 2.5 times the previously claimed uncertainties. Therefore, this consistency factor was used in the 1995 adjustment [7] for increasing the uncertainties of these input data, instead of the factor of 1.5 already applied in the 1993 mass evaluation [25].

\subsection{Comparison of ISOLTRAP data with adjusted results}

The adjusted results [7] are given in column 5 of Table 5 . In addition, the sum of influences $I F$ brought by the Penning trap data is given in column 6 . This value is the percentage of the contribution of these data [35] to the determination of the mass values in the adjustment. From Table 5 it can be seen that in 18 cases of the 41 measured masses the influence of the ISOLTRAP data is greater than 50\%, increasing to 21 cases when the not yet adjusted masses of the three light odd barium isotopes are taken into account. For 8 isotopes it is between $20 \%$ and $50 \%$. The remaining ones include eight measured values which have no influence on the global adjustment. Nevertheless these ISOLTRAP data agree very well with the adjusted values determined by other very accurate mass measurements. This confirms our assumption on the maximum systematic error of $1 \cdot 10^{-7}$ during on-line measurements.

It is interesting to note the effect of the ISOLTRAP measurements on the nuclide ${ }^{133} \mathrm{Cs}$ used here as a reference isotope. Table 5 shows that its precision is significantly improved by the present data: as much as $45 \%$ of the information on its adjusted mass value is derived from the ISOLTRAP measurements. Therefore, the mass values given in Table 5, which have been obtained via Eqn. 3, should always be quoted together with the mass value used for the ${ }^{133}$ Cs-reference.

The impact of the ISOLTRAP data on the recent atomic mass 1995 update [7] is directly visualized in Fig. 5 and 6 by comparing the plots in the upper 
(1986 mass adjustment, without ISOLTRAP data) with the lower part (1995 mass adjustment, with ISOLTRAP data). The adjusted values represent the zero line and their errors are given as an error band. The data points are the difference between the direct Penning trap mass values (Table 5) and the adjusted mass values of all available and accepted mass data. Note again that the isotopes ${ }^{123,125,127,131} \mathrm{Ba}$ published recently [4] are not included in the 1995 mass update.

It is clear that the errors for the neutron-deficient cesium isotopes and the neutron-rich barium isotopes have been significantly reduced and that a very consistent picture of the mass surface in this region has resulted. This is due to the very high and consistant accuracy of the ISOLTRAP data, even for isotopes far from stability, and demonstrates the high reliability of the data obtained by Penning trap mass spectrometry.

\subsection{Comparison with mass formulae}

All nuclear models and mass formulae rely on input from experiment: The experimental data serve to adjust the parameters of the models and at the same time to check the results of the calculations. Figure 7 shows as an example the comparison of the data presented in this paper with some current mass formulae.

In the top of Fig. 7 the difference is given between experimental and calculated masses for the model of Jänecke and Masson [36]. This mass formula belongs to a class of models based on the so-called Garvey-Kelson relations [37]. Due to its very strong connection with experimental values in an extreme single particle picture this model reproduces most successfully the experimental masses but it has a very limited predictive power for nuclei far from stability [38]. In fact, a significant systematic deviation between theory and experiment is observed only for the very neutron-rich cesium and barium isotopes.

The next set of pictures shows the differences between the experimental masses and those calculated by a new macroscopic-microscopic parametrization [39] with only 10 parameters that can easily be calculated. In spite of its simplicity it gives satisfying results except for a large odd-even staggering for the neutronrich cesium and proton-rich barium isotopes.

The third set of pictures in Fig. 7 shows the comparison with the macroscopicmicroscopic model of Möller and Nix [40,41]. This model is a combination of a single-particle shell-model based on a folded-Yukawa potential and a collective liquid-drop or droplet model. From the plot in Fig. 7 it can be seen that the predicted values deviate not in a statistic but in a systematic manner from the measurement. Especially significant deviations are observed close to the 
shell closure at $\mathrm{N}=82$.

The plots shown at the bottom of Fig. 7 compare the experimental masses with the extended Thomas-Fermi model of Pearson et al. [42]. This model avoids the arbitrary division in a macroscopic and a microscopic part by calculating both gross and single particle effects from the same Skyrme force. The authors of this model admit that it is not yet in its final state, but its global agreement with experimental masses is nearly as good as that of the macroscopic-microscopic models. In the cases of cesium and barium the effect of the shell closure at $\mathrm{N}=82$ is again overestimated, the treatment of the pairing of nucleons shows deficiencies for nearly all neutron-rich cesium isotopes and rather strong discrepancies are observed for the most neutron deficient cesium as well as for the most neutron-rich cesium and barium isotopes.

In [38] a comparison of the predictive power of different nuclear models was performed by comparing their predictions of nuclear masses unknown at the time of calculation with the results obtained in later experiments. Not surprisingly, the models of the Garvey-Kelson type were attested to deliver poor predictions, whereas the models with a more firm physical basis keep their admittedly larger errors constant when extrapolating to more exotic nuclei.

\subsection{A macroscopic-microscopic mass formula - prediction of masses and de- formations}

The models of the macroscopic-microscopic type combine a liquid-drop model and a shell model of the nucleus. While the first one describes the general behaviour of nuclear properties (varying smoothly with $\mathrm{N}$ and $\mathrm{Z}$ ), the second one introduces the quantum-mechanical behaviour of nuclear shells with shell corrections. The characteristic odd-even variations of nuclear properties are accounted for by a pairing correction. The shell corrections are usually calculated according to the Strutinsky prescription [43] and pairing corrections in the spirit of the BCS theory of superconductivity. The single-particle energies calculated from a given nuclear potential serve as an input to both corrections.

We have performed calculations in the frame of such a model. Our aim was to investigate the quality of the model predictions that can be achieved if only a local adjustment of selected model parameters is performed. Similar calculations to those presented here have already been performed for rubidium and strontium isotopes and are discussed in detail in Ref. [3]. A brief discussion of the investigations for these isotopes and for those of cesium and barium has also been given in [44]. Therefore, we will summarise the principles of these calculations and describe particular details of the calculations on cesium and barium isotopes. 
For the macroscopic part of the model we use the Yukawa-plus-exponential model [45] in the specific form of [46]. The microscopic part is based on the deformed Woods-Saxon single-particle potential [47]. We assume only axiallysymmetric deformations of the nucleus and develop the nuclear surface in terms of multipoles with deformation parameters $\beta_{\lambda}$. The determination of the minimum of the total potential energy in a sufficiently large deformation space $\left\{\beta_{\lambda}\right\}$ gives the equilibrium deformation of the nucleus. In view of the expected octupole deformation of the neutron-rich Cs and Ba isotopes [48,49] a six-dimensional deformation space $\left\{\beta_{\lambda}\right\}, \lambda=2,3,4,5,6,7$ has been chosen. The residual pairing interaction is treated in the Lipkin-Nogami approach [50]. Our calculations proceed as follows: For every nucleus considered we determine the equilibrium deformation from the shape-dependent parts of our mass formula, assuming certain values for the two free parameters $r$ and $t$ of the effective pairing gap [40]. As a next step we adjust the volume asymmetry parameter $\kappa_{v}$, the charge asymmetry parameter $c_{a}$ and the overall parameter $c_{0}$ of the macroscopic part [46] to obtain the smallest root-mean-square (RMS) deviation between the theoretical and all known experimental masses in one isotopic chain. The values of all other macroscopic parameters are taken from [46] and are held fixed. The two steps - the finding of the equilibrium deformation and the adjustment of the macroscopic parameters - are consecutively repeated for different sets of the pairing parameters $r$ and $t$ until a minimum RMS deviation from the experimental mass values is achieved.

The result of this local adjustment is shown in Tab. 6. The values of the macroscopic parameters $\kappa_{v}, c_{a}$ and $c_{0}$ are in good agreement with those found in [46], where a global adjustment of the model parameters was performed, and with the ones found in a mass calculation in the heavy-nuclei region [51]. The response of the model to a change of the pairing parameter $t$ was found to be soft. The parameter $r$ is of the same order of magnitude as that obtained by Möller and Nix in their global adjustment [40].

Our calculations confirmed the existence of odd-multipole deformations for the neutron-rich $\mathrm{Cs}(\mathrm{N}=85-94)$ and $\mathrm{Ba}(\mathrm{N}=86-92)$ isotopes. The values of $\beta$-deformations for even-even $\mathrm{Ba}$ isotopes are (except for $\mathrm{N}=94$ ) in agreement with [48 $]^{10}$ and for odd-A Cs and Ba isotopes with [49] ${ }^{11}$.

Figure 8 gives a comparison of the theoretical mass values with the experimental ones. The deviations from experiment exhibit a regular behaviour. The main source of these deviations lies probably in both pairing and shell corrections. It is seen that in the vicinity of $\mathrm{N}=82$ the discrepancy between theory and experiment is increasing, probably due to the deficiency of the pairing correction close to magic numbers. The model description is also worse for the

\footnotetext{
$\overline{10}$ calculation for even-even nuclei only

11 calculation for odd-A nuclei only
} 
lightest isotope of cesium, which might be due to the one-particle instability of this nucleus. The present results $(\mathrm{RMS}(\mathrm{Cs})=238 \mathrm{keV}, \mathrm{RMS}(\mathrm{Ba})=227$ $\mathrm{keV})$ are in a better agreement with experiment than those of comparable approaches shown in the lower part of Figure 7. This is not too surprising since the local adjustment is restricted to one isotopic chain and a larger deformation space is used here.

Extending the analysis of nuclear ground state properties, we would like to compare the nuclear quadrupole deformation values $\beta_{2}^{\text {th }}$, as predicited by the locally adjusted model, with those deduced from other experiments. For the isotopic chains discussed here, data are available from optical isotopic shift measurements (IS) from which a deformation parameter $\beta_{2}^{I S}$ can be extracted. Further information is provided by hyperfine structure measurements, from which spectroscopic quadrupole moments $Q_{s}$ and from these $\beta_{2}^{Q}$ can be obtained.

The $\beta_{2}^{\text {th }}$ values result from the minimisation procedure of the total nuclear energy as discussed above. The values for $\beta_{2}^{I S}$ have been re-calculated in a uniform way from the mean-square nuclear charge radii differences $\delta\left\langle r^{2}\right\rangle$ obtained from isotope shift measurements and the values of $\beta_{2}^{Q}$ from the spectroscopic quadrupole moments $Q_{s}$ presented in Refs. [52], [53], [54] and [55]. The mean-square charge radii differences $\delta\left\langle r^{2}\right\rangle$ were corrected for the contribution of higher radial moments according to the prescription given in [56].

We follow the generally adopted procedure of extracting $\beta_{2}^{I S}$ from $\delta\left\langle r^{2}\right\rangle$, using the relation

$$
\left\langle r^{2}\right\rangle=\left\langle r^{2}\right\rangle_{s p h} \cdot\left(1+\frac{5}{4 \pi}\left\langle\beta_{2}^{2}\right\rangle_{I S}\right)
$$

between the mean-square charge radius $\delta\left\langle r^{2}\right\rangle$ and the quadrupole deformation parameter $\beta_{2}^{I S}$ of the nuclear charge distribution. Here $\left\langle r^{2}\right\rangle_{s p h}$ is the mean square radius of a spherical nucleus with the same volume as the deformed nucleus. Calculating $\left\langle r^{2}\right\rangle_{s p h}$ in the droplet model of Myers [57] and using (4) one obtains $\beta_{2}^{I S}=\left\langle\beta_{2}^{2}\right\rangle_{I S}^{1 / 2}$ as a measure of the static as well as the dynamic deformation.

The intrinsic nuclear quadrupole moment $Q_{0}$ is related to the spectroscopic moment $Q_{s}$ for axially symmetric nuclei in the strong coupling limit by

$$
Q_{0}=\frac{(I+1)(2 I+3)}{I(2 I-1)} Q_{s}
$$

where $I$ is the spin of the nucleus. Then, from $Q_{0}$ we deduce the mean defor- 
mation $\beta_{2}^{Q}$ using the formula [58]

$$
Q_{0}=\frac{3}{\sqrt{5 \pi}} Z r_{0}^{2} A^{2 / 3}\left\langle\beta_{2}^{Q}\right\rangle\left(1+\frac{2}{7} \sqrt{\frac{5}{\pi}}\left\langle\beta_{2}^{Q}\right\rangle\right), \quad r_{0}=1.2 \mathrm{fm},
$$

where $Z$ denotes the atomic and $A$ the mass number. This formula corresponds also to the second-order expansion of the exact macroscopic (volume integral) quadrupole moment expression calculated with the same nuclear radius parametrization as used in our mass calculations. Obtaining $\beta_{2}^{I S}$ and $\beta_{2}^{Q}$ as described above is of course model dependent.

As already stated, the $\beta$-information obtained from $I S$ and $Q_{s}$ is the measure of deformation of the nuclear charge distribution, whereas $\beta_{2}^{\text {th }}$ corresponds to the quadrupole deformation of the nuclear potential. It has been shown in [59] that for the Woods-Saxon potential used in the present calculations one should multiply $\beta_{2}^{\text {th }}$ by a factor of 1.1 before comparing it with $\beta_{2}^{I S}$ and $\beta_{2}^{Q}$ deformations. Furthermore, since $\beta_{2}^{I S}$ is lacking a sign, we take the modulus of the theoretical $\beta_{2}$ and the experimental $\beta_{2}^{Q}$-values for comparison. Such a comparison is shown in Fig. 9, where $\beta_{2}^{\text {th }} \cdot 1.1, \beta_{2}^{I S}$ and $\beta_{2}^{Q}$ are plotted as a function of $N$. In order to keep the sign information the symbols for $\beta_{2}^{\text {th }}$ and $\beta_{2}^{Q}$ are marked accordingly.

As expected the deformation parameters derived from the microscopic-macroscopic model by fitting the experimental masses and the deformation parameters extracted from the $I S$ and $Q_{s}$ data agree well for strongly deformed nuclei. For transitional nuclei and those near the shell closure at $\mathrm{N}=82$ the $\beta_{2}^{I S}$ values are generally larger than $\beta_{2}^{\text {th }}$ and $\beta_{2}^{Q}$. For the Ba isotopes, the $\beta_{2}^{Q}$-values agree with $\beta_{2}^{\text {th }}$ fairly well. This is not always the case for Cs nuclei, where the isotopes with $\mathrm{N}=69,71$ and 73 have $\beta_{2}^{Q}$ values greater than 0.4 (not included in Fig. 9) and the $\beta_{2}^{Q}$-values are in several cases much smaller than $\beta_{2}^{\text {th }}$ and $\beta_{2}^{I S}$. A closer look shows that in all these cases $\beta_{2}^{Q}<0$.

The behaviour shown in Fig. 9 can be understood qualitatively: Whereas $\beta_{2}^{\text {th }}$ and $\beta_{2}^{Q}$ are measures for static deformations, $\beta_{2}^{I S}$ is also sensitive to vibrations leading to a larger deformation parameter deduced from IS measurements. All deformation parameters should approach each other with the increase of deformation, where the relative influence of the vibrations should fade out. Furthermore, $\beta_{2}^{Q}$ calculated under the assumption of strong coupling underestimates the "true" deformation in the case of transitional nuclei, as can be clearly seen in the example of $I=13 / 2$ isomers of mercury [60]. 


\section{Conclusions}

High-accuracy mass measurements on $\mathrm{Cs}$ and $\mathrm{Ba}$ isotopes have been performed with the ISOLTRAP mass spectrometer at the on-line mass separators ISOLDE-II and PSB-ISOLDE. The masses of 41 isotopes were determined including the masses of ${ }^{122} \mathrm{Cs}^{m}$ and ${ }^{123,124,126} \mathrm{Ba}$ which were measured for the first time. For these isotopes an accuracy of the mass measurements of about $1 \cdot 10^{-7}$ corresponding to $14 \mathrm{keV}$ was achieved. The spectrometer was operated with a resolving power of typically 600000 due to the limited beam time available for these on-line experiments. In off-line measurements a resolving power of $\approx 8000000$ was obtained in this mass region.

The data of the ISOLTRAP spectrometer have been used for several leastsquares adjustment of all available mass data. During this procedure a number of wrong mass values have been found in the 1986 mass tables [24]. The uncertainties of the mass values especially for isotopes far off stability could be significantly reduced due to the high accuracy of the Penning trap data.

The comparison of the experimental data with the predictions of mass formulae shows disagreements exceeding the experimental errors by up to two orders of magnitude. A better agreement could be achieved by adjusting the parameters of a macroscopic-microscopic model locally for one isotopic chain. For strongly deformed nuclei the quadrupole deformation parameters $\beta_{2}$ deduced from the macroscopic-microscopic model used for fitting the mass values are in a good agreement with those from isotope shift data and from spectroscopic quadrupole moments. For transitional nuclei discrepancies are observed which are not surprising. A lot of work is still needed on the theoretical side to close the gap between mass predictions and experimental values.

\section{Acknowledgement}

The authors are indebted to A. Sobiczewski for valuable comments concerning the theoretical part. We acknowledge financial support by the Bundesministerium für Forschung und Technologie under Contract Hz188-I and by NSERC of Canada. Travel was also supported by NATO grant no. CRG 900675. The work of J. Szerypo was partially supported by KBN contract no. PB 202089101 . Z. Patyk acknowledges generous support by CSNSM, University of Mainz and the Polish State Committee for Scientific Research grant no. 209549101. 


\section{A Appendix}

The physical quantities measured in this experiment are ratios

$$
r=\frac{\nu_{r e f}}{\nu}
$$

of the cyclotron frequency $\nu_{\text {ref }}$ of the reference isotope and the cyclotron frequency $\nu$ of the investigated isotope. These frequency ratios correspond to the mass ratio of ionic species

$$
r=\frac{m-m_{e}}{m_{r e f}-m_{e}}
$$

where $m$ and $m_{\text {ref }}$ denote the atomic mass of the investigated and reference isotope and $m_{e}$ is the mass of the electron. The binding energy of the valence electron can be neglected.

In order to use the results of this work in the standard least-squares method for the evaluation of masses [61], the frequency ratios $r$ have to be recast into linear relations between the mass excess of the unknown and the reference isotope. Equation (A.2) can be written in the form

$$
M E-r \cdot M E_{r e f}=m_{e}(1-r)+A_{r e f}\left(r-\frac{A}{A_{r e f}}\right)
$$

with $M E$ and $M E_{\text {ref }}$ denoting the mass excess and $A$ and $A_{r e f}$ the mass number of the isotope with unknown mass and the reference isotope (all masses are expressed in atomic mass units). We now define $\mathrm{C}$ as a rounded three-digit approximation of $A$ over $A_{\text {ref }}$

$$
C=\left(\frac{A}{A_{\text {ref }}}\right)_{\text {rounded to three digits }} .
$$

This leads to the desired result

$$
M E-C \cdot M E_{\text {ref }}=M D
$$

with

$$
M D=M E_{r e f}(r-C)+m_{e}(1-r)+A_{r e f}\left(r-\frac{A}{A_{r e f}}\right)
$$


The averaged frequency ratios in Table 3 (run \#1 - \#4) and those in Table 4 (run \#5 and \#6) are converted in Table 7 and Table 8 into linear relations between atomic mass excesses in order to derive adjusted masses. These equations have been added to the ensemble of equations obtained from reaction data, decay energies, or other mass spectrometric data.

In performing preliminary least-squares adjustments, several discrepancies were observed as discussed in Section 6. They were subsequently corrected or taken out by the evaluators of atomic masses in their 1993 mass tables [25] and the 1995 update [7].

In the 1993 atomic mass evaluation [25], the results of runs \#1 - \#4 (Tables 3 and 7) were included. For the 1995 mass update [7] the 10 data of runs \#5 and \#6 given in Tables 4 and 8 were added. Also the new estimated isomeric excitation energies discussed above have replaced the older ones.

The results from the least-squares fit on this set of data are given in column 5 of Table 5 for the adjusted mass values and in column 3 of Tables 7 and 8 for the adjusted ISOLTRAP equations. Where no value is given in the latter (equations involving ${ }^{117} \mathrm{Cs}$ and ${ }^{124,126} \mathrm{Ba}$ ) no adjustment is necessary and the output value is identical to the input one ("secondary" data). Column 4 in Tables 7 and 8 gives the consistency of the ISOLTRAP data with the adjustment, defined as the ratio of the deviation $v$ of the trap data from the adjusted values divided by the uncertainty $s$ of the Penning trap values.

The significances of the ISOLTRAP data, or total influence of these data on all masses are given in the last columns of Tables 7 and 8. The "secondary" data involving ${ }^{122 m} \mathrm{Cs}$ (as an average of 2 equations appearing one in table 7 , the other in table 8), ${ }^{117} \mathrm{Cs}^{x}$ and ${ }^{124,126} \mathrm{Ba}$ have $100 \%$ significancy and each of these nuclides receives $100 \%$ influence from the corresponding datum. 


\section{References}

[1] G. Bollen, Nucl. Phys. A626 (1997) 297c

[2] H. Stolzenberg, St. Becker, G. Bollen, F. Kern H.-J. Kluge, Th. Otto, G. Savard, L. Schweikhard, G. Audi and R.B. Moore, Phys. Rev. Lett. 65 (1990) 3104

[3] T. Otto, G. Bollen, G. Savard, L. Schweikhard, H. Stolzenberg, G. Audi, R. B. Moore, G. Rouleau, J. Szerypo and Z. Patyk, Nucl. Phys. A567 (1994) 281

[4] D. Beck, F. Ames, G. Audi, G. Bollen, H.-J. Kluge, A. Kohl, M. König, D. Lunney, I. Martel, R.B. Moore, H. Raimbault-Hartmann, E. Schark, S. Schwarz, M. de Saint Simon, J. Szerypo, and the ISOLDE Collaboration, Nucl. Phys. A626 (1997) 343c

[5] G. Bollen, H.-J. Kluge, Th. Otto, G. Savard, L. Schweikhard, H. Stolzenberg, G. Audi, R.B. Moore and G. Rouleau, J. Modern Optics 39 (1992) 257

[6] S. Schwarz et al., in preparation

[7] G. Audi and A.H. Wapstra, Nucl. Phys. A595 (1995) 409

[8] H. G. Dehmelt, Adv. At. and Mol. Phys. 3 (1967) 53

[9] H. G. Dehmelt, Adv. At. and Mol. Phys. 5 (1969) 109

[10] D. J. Wineland, W. M. Itano and R. S. Van Dyck Jr., Adv. At. and Mol. Phys. 19 (1983) 135

[11] L. S. Brown and G. Gabrielse, Rev. Mod. Phys. 58 (1986) 233

[12] G. Bollen, R. B. Moore, G. Savard and H. Stolzenberg, J. Appl. Phys. 68 (1990) 4355

[13] M. König, G. Bollen, H.-J. Kluge, T. Otto and J. Szerypo, Int. J. Mass Spectr. Ion. Proc. 142 (1995) 95

[14] G. Gräff, H. Kalinowsky and J. Traut, Z. Phys. A297 (1980) 135

[15] E. Kugler, D. Fiander, B. Jonson, H. Haas, A. Przewloka, H.L. Ravn, D.J. Simon, K. Zimmer, and the ISOLDE Collaboration, Nucl. Instr. and Meth. B70 (1992) 41

[16] G. Bollen, S. Becker, H.-J. Kluge, M. König, R.B. Moore, T. Otto, H. Raimbault-Hartmann, G. Savard, L. Schweikhard, H. Stolzenberg, and the ISOLDE Collaboration, Nucl. Instr. Meth. A368 (1996) 675

[17] H. Raimbault-Hartmann, D. Beck, G. Bollen, M. König, H.-J. Kluge, E. Schark, J. Stein, S. Schwarz, and J. Szerypo, Nucl. Instr. and Meth. B126 (1997) 378

[18] G. Savard, St. Becker, G. Bollen, H.-J. Kluge, R. B. Moore, Th. Otto, L. Schweikhard, H. Stolzenberg and U. Wiess, Phys. Lett. A158 (1991) 247 
[19] H. Schnatz, G. Bollen, P. Dabkiewicz, P. Egelhof, F. Kern, H. Kalinowsky, L. Schweikhard, H. Stolzenberg and H.-J. Kluge, Nucl. Instr. Meth. A251 (1986) 17

[20] G. Bollen, H.-J. Kluge, M. König, T. Otto, G. Savard, H. Stolzenberg, R. B. Moore, G. Rouleau and G. Audi, Phys. Rev. C46 (1992) R2140

[21] K. Kitao, M. Kanbe, Z. Matsumoto and T. Seo, Nuclear Data Sheets 39 (1986), 315

[22] G. Audi, M. Epherre, C. Thibault, A. H. Wapstra and K. Bos, Nucl. Phys. A378 (1982) 443

[23] G. Audi et al., Nucl. Phys. A624 (1997) 1

[24] A. H. Wapstra, G. Audi and R. Hoekstra, At. Data. Nucl. Data Tables 39 (1988) 290

[25] G. Audi and A.H. Wapstra, Nucl. Phys. A565 (1993) 1, and G. Audi, A.H. Wapstra and M. Dedieu, Nucl. Phys. A565 (1993) 193

[26] L. Lidofsky, E. Alperovitch and C. S. Wu, Phys. Rev. 90 (1953) 387

[27] S. Ulbig (1992), priv. comm. to A.H. Wapstra, in: G. Audi, A.H. Wapstra and M. Dedieu, Nucl. Phys. A565 (1993) 193

[28] A. B. Smith, A. C. G. Mitchell and R. S. Caird, Phys. Rev. 87 (1952) 454

[29] P. Debenham, W. R. Falk and M. Canty, Can. J. Phys. 52 (1974) 1416

[30] A. Blönnigen, Diploma work, Giessen (1980) unpublished

[31] M. Przewloka, A. Przewloka, P. Wächter and H. Wollnik, Z. Phys. A342 (1992) 27

[32] D. W. Hetherington, PhD thesis, Montreal (1984), unpublished

[33] G. Audi, A. Coc, M. Epherre-Rey-Campagnolle, G. le Scornet, C. Thibault and F. Touchard, Nucl. Phys. A 449 (1986) 491

[34] G.D. Alkhazov, B.N. Belyayev, V.D. Domkin, Yu.G. Korobulin, V.V. Lukashevich and V.S. Mukhin, Iz. Ak. Nauk 53 (1989) 2089

[35] G. Audi, W.G. Davies and G.E. Lee-Whiting, Nucl. Instr. Meth. A249 (1986) 443

[36] J. Jänecke and P. J. Masson, At. Data Nucl. Data Tables 39 (1988) 265

[37] G. T. Garvey and I. Kelson, Phys. Rev. Lett. 16 (1966) 197

[38] C. Borcea and G. Audi, Roumanian J. Phys. 48 (1993) 445; CSNSM-9238 report, Orsay 1992.

[39] J. Duflo Nucl. Phys.A576 (1992) 29

[40] P. Möller and J.R. Nix, Nucl. Phys. A536 (1992) 20 
[41] P. Möller, J.R. Nix, W. D. Myers and W. J. Swiatecki, Nucl. Phys. A536 (1992) 61

[42] J.M. Pearson, Y. Aboussir, A.K. Dutta, R.C. Nayak and M. Farine, Nucl. Phys. A528 (1991) 1

[43] V.M. Struntinsky, Nucl. Phys. A122 (1986) 1

[44] J. Szerypo and Z. Patyk, Proc. 6th Int. Conf. on Nuclei far from Stability\& 9th Int. Conf. on At. Masses and Fund. Constants, Bernkastel-Kues, 1992, Inst. Phys. Conf. Ser. No 132 (1993) 85

[45] H. J. Krappe, R. Nix and A. J. Sierk, Phys. Rev. C20 (1979) 992

[46] P. Möller and J.R. Nix, At. Data Nucl. Data Tables 26 (1981) 165

[47] S. Ćwiok, J. Dudek, W. Nazarewicz, J. Skalski and T. Werner, Comp. Phys. Comm. 46 (1987) 379

[48] A. Sobiczewski, Z. Patyk, S. Ćwiok and P. Rozmej, Nucl. Phys. A485 (1988) 16

[49] S. Ćwiok and W. Nazarewicz, Nucl. Phys. A469 (1989) 367

[50] H.C. Pradhan, Y. Nogami and J. Law, Nucl. Phys. A201 (1973) 357

[51] Z. Patyk and A. Sobiczewski, Nucl. Phys. A533 (1991) 132

[52] C. Thibault, F. Touchard, S. Büttgenbach, R. Klapisch M. de Saint Simon, H. T. Duong, P. Jacquinot, P. Juncar, S. Liberman P. Pillet, J. Pinard, J. L. Vialle, A. Pesnelle and G. Huber, Nucl. Phys. A367 (1981) 12

[53] A. Coc, C. Thibault, F. Touchard, H. T. Duong, P. Juncar, S. Liberman, J. Pinard, M. Carre, J. Lerme, J. L. Vialle, S. Büttgenbach, A. C. Mueller and A. Pesnelle, Nucl. Phys. A468 (1987) 1

[54] A. C. Mueller, F. Buchinger, W. Klempt, E. W. Otten, R. Neugart, C. Ekström and J. Heinemeier, Nucl. Phys. A403 (1983) 234

[55] K. Wendt, S. A. Ahmad, C. Ekström, W. Klempt, R. Neugart and E. W. Otten, Z. Phys. A329 (1988) 407

[56] S. A. Ahmad, W. Klempt, R. Neugart, E. W. Otten, P.-G. Reinhard, G. Ulm and K. Wendt, Nucl. Phys. A483 (1988) 244

[57] W. D. Myers, Droplet model of atomic nuclei, (Plenum, New York, 1977)

[58] K. E. G. Löbner, M. Vetter and V. Hönig, Nucl. Data Tables A7 (1970) 495

[59] W. Nazarewicz, M. A. Riley and J. D. Garrett, Nucl. Phys. A512 (1990) 61

[60] H. Kremmling, P. Dabkiewicz, H. Fischer, H.-J. Kluge, T. Kühl and H.A. Schüssler, Phys. Rev. Lett. 43 (1979) 1376

[61] A. H. Wapstra and G. Audi, Nucl. Phys. A432 (1985) 1 


\section{Figure 1}

Experimental setup of the ISOLTRAP mass spectrometer as it was installed at the ISOLDE-II and PS-Booster ISOLDE on-line isotope separator at CERN up to 1994. The lower part shows the first Penning trap (used for cooling and bunched ejection of the radioactive isotope under investigation) in an electro magnet. The precision trap applied for mass determination (upper part) is installed in a super-conducting solenoid. The ion detector on top of the apparatus is used for the time-of-flight detection of the cyclotron resonances. In 1995, the electromagnet was replaced by a superconducting magnet [17].

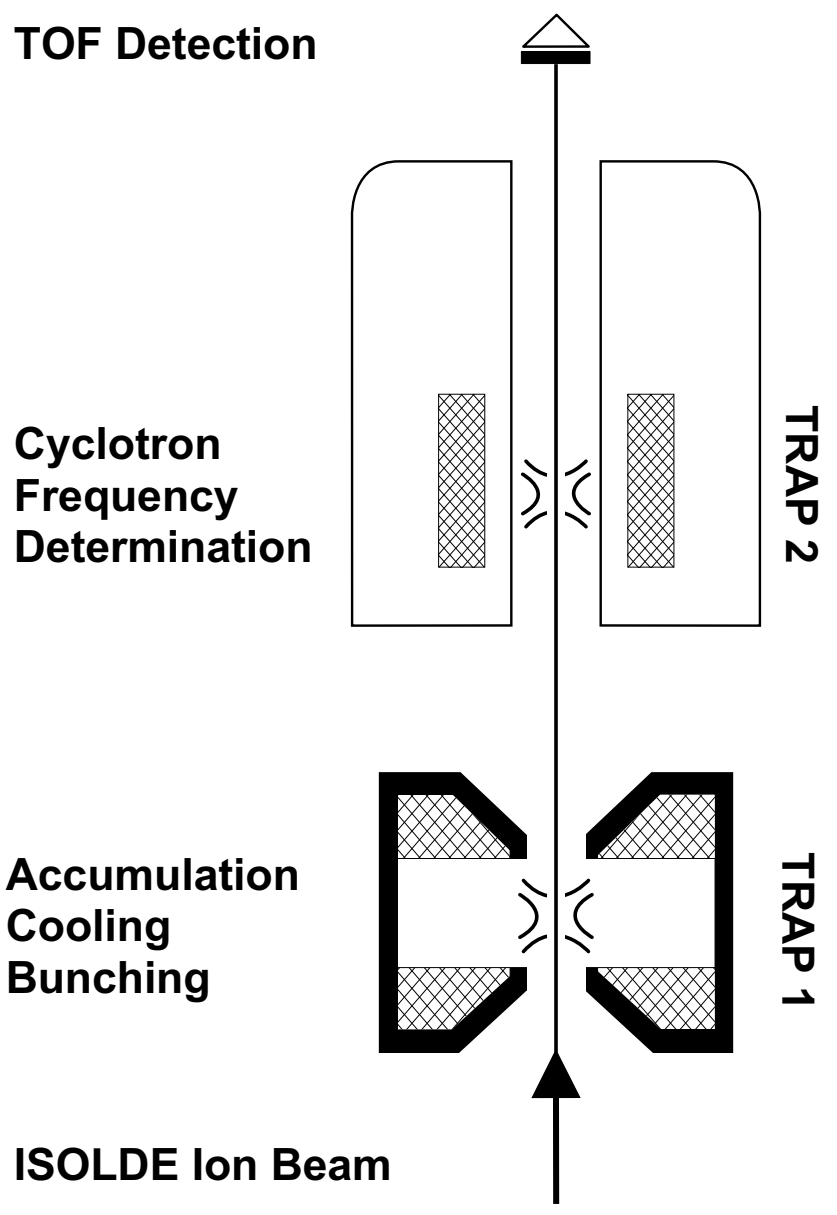




\section{Figure 2}

Cyclotron resonance of ${ }^{118} \mathrm{Cs}$ as obtained from the mean time of flight of the ions from the trap to the detector as a function of the frequency of the $\mathrm{RF}$ field. The resolving power $R=\nu_{c} / \Delta \nu_{c}(F W H M)$ of this measurement is $R \approx 640000$. The solid line is a fit of the data points by a Gaussian.

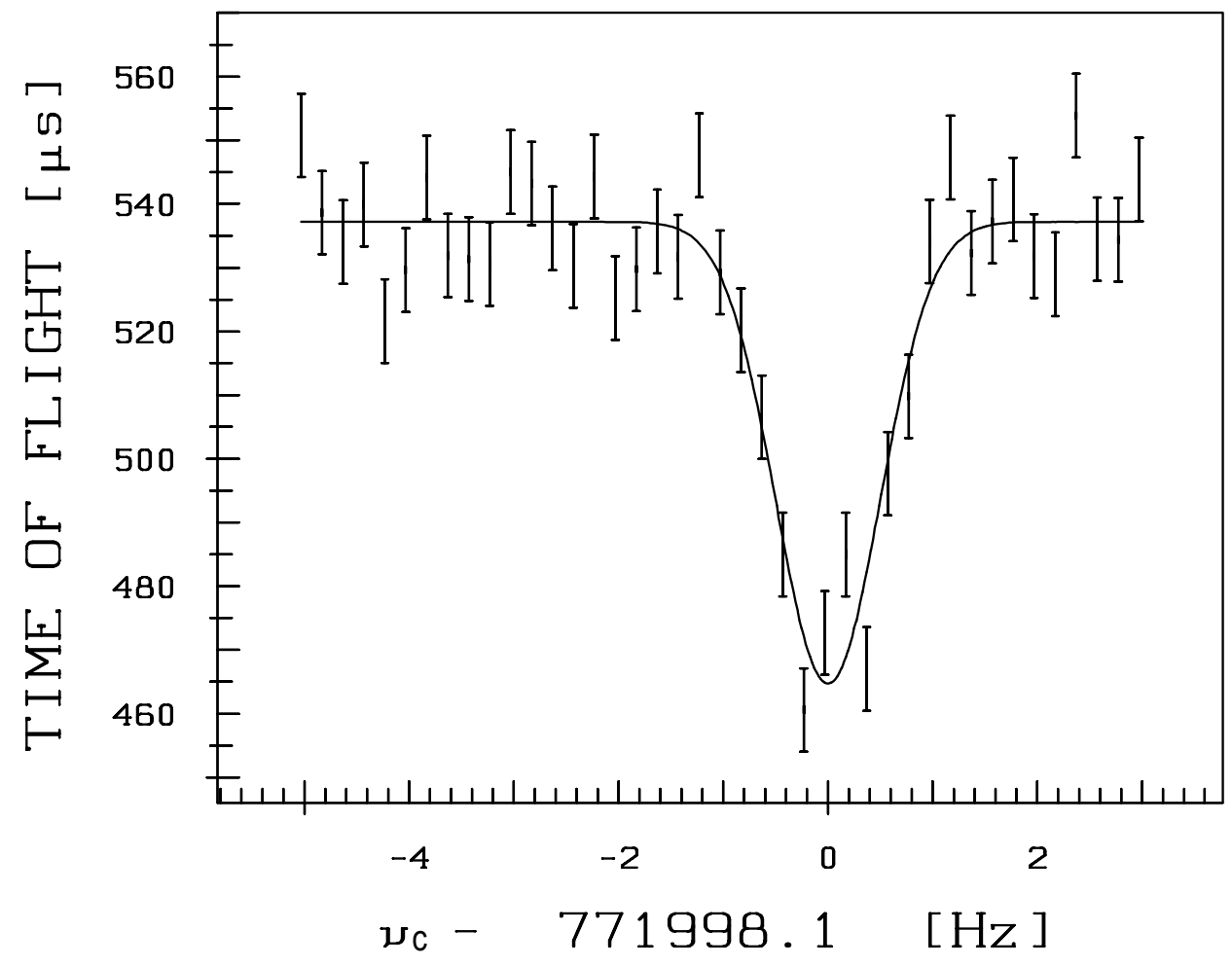




\section{Figure 3}

Cyclotron resonances of ${ }^{122} \mathrm{Cs}$ which has two long-lived nuclear states with $T_{1 / 2}=3.7 \mathrm{~min}$ and $T_{1 / 2}=21 \mathrm{~s}$. Top: Resonance of the pure $\left(T_{1 / 2}=3.7 \mathrm{~min}\right)$ component. Bottom: Resonance obtained with nuclei in both states stored simultaneously in the trap. Note that the line width (FWHM) and the reduction of the time of flight in resonance (TOF) are identical in both cases.

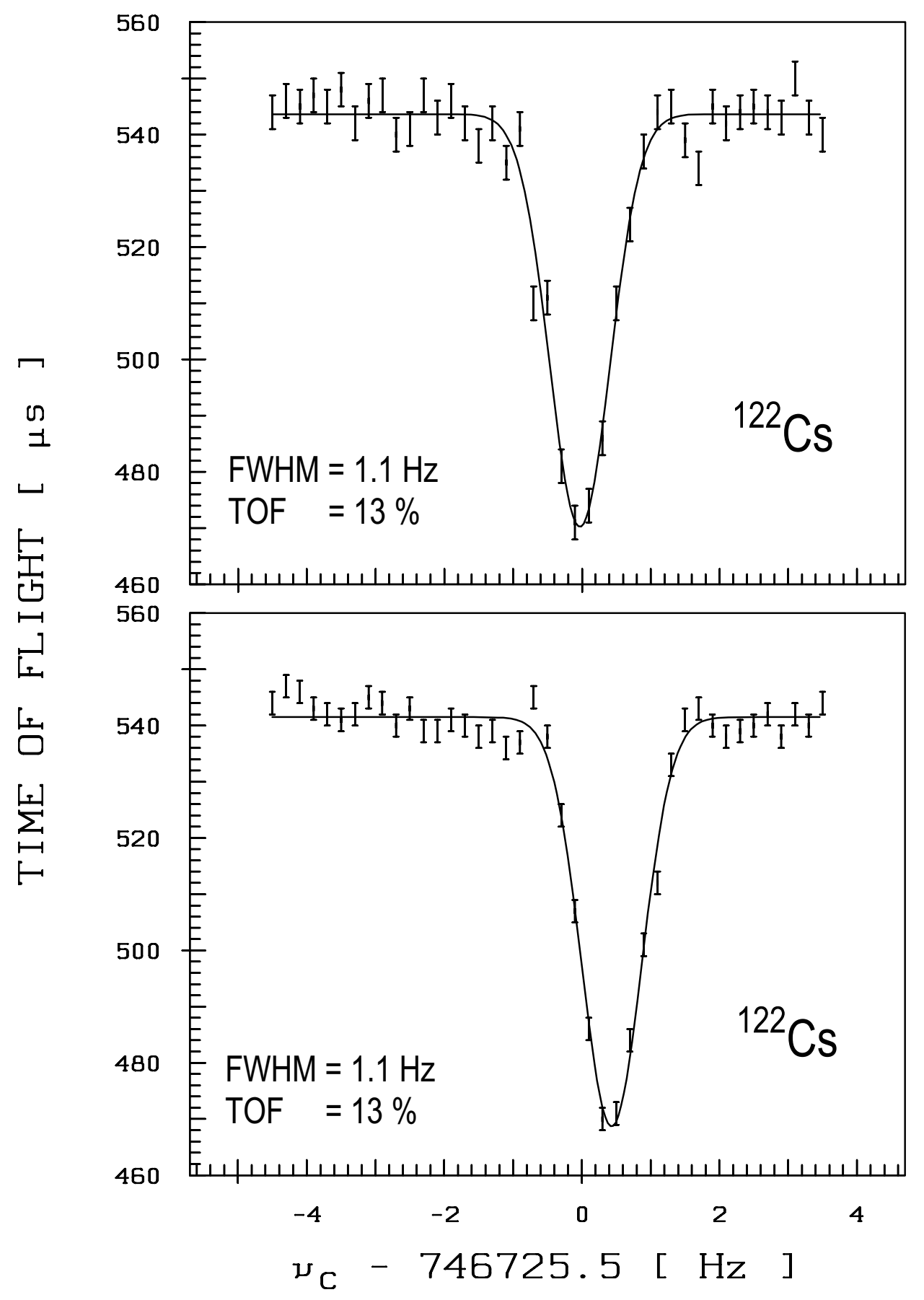




\section{Figure 4}

Shift of the center frequency of the cyclotron resonance for a mixture of ${ }^{122} \mathrm{Cs}$ and ${ }^{122 m} \mathrm{Cs}$ as a function of the delay between collection of the sample and start of the measurement. $\nu_{c}\left(T_{1 / 2}=3.7 \mathrm{~min}\right)$ is the cyclotron frequency of the pure long-lived component. The dashed line represents a fit to the data yielding an excitation energy of the isomer of $135(14) \mathrm{keV}$, and a ratio of $R_{m g}=0.9(2)$ for the population of the isomeric relative to the ground state. In this way the $21 \mathrm{~s}$ half-life state is determined to be the ground state.

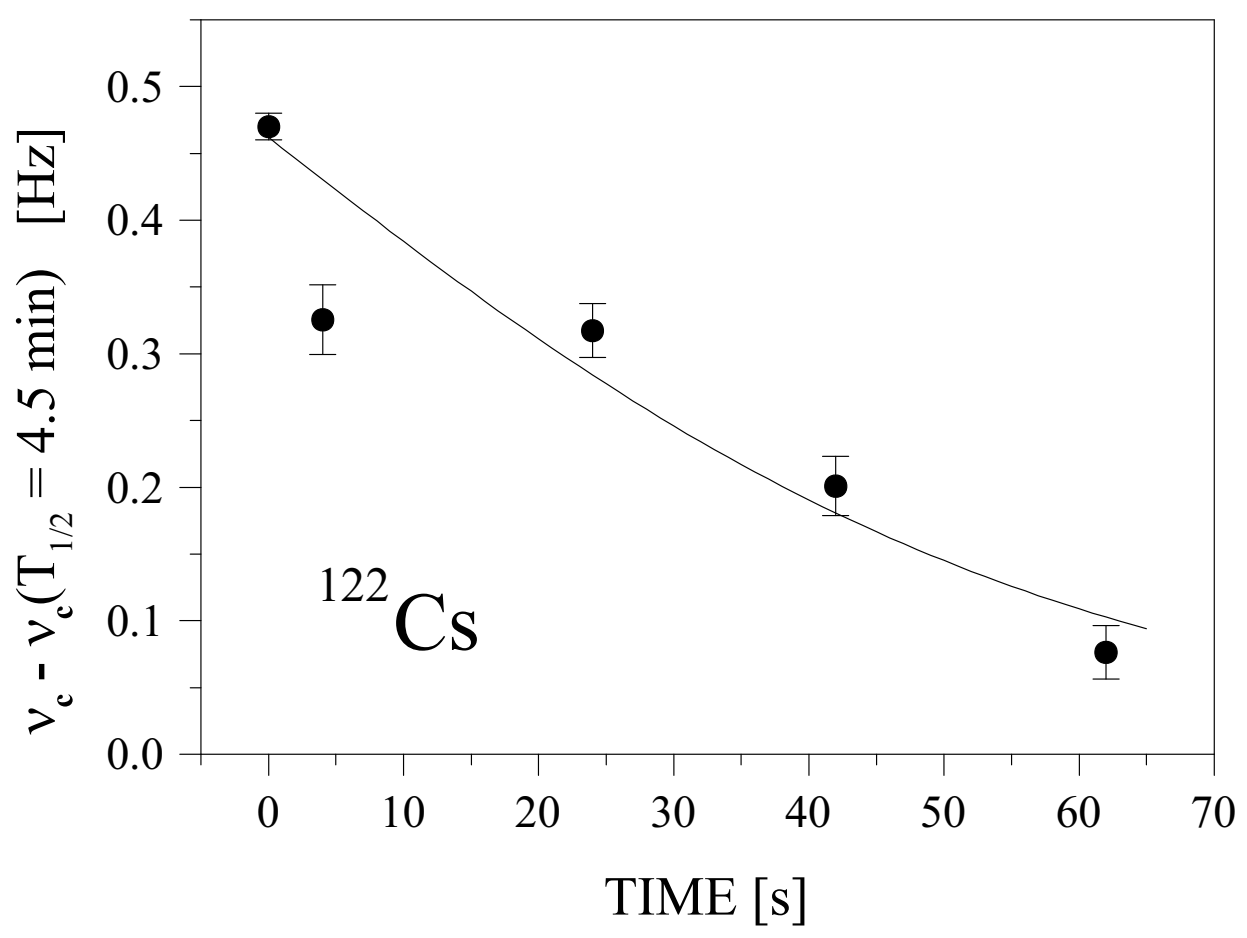




\section{Figure 5}

Comparison of the masses for cesium isotopes as calculated from the cyclotron frequencies with the adjusted masses. The difference is plotted versus the mass number. The adjusted masses represent the zero line and their uncertainties are indicated by the error band. The error bars of the data points are the uncertainties of the Penning trap mass measurements. Top: difference relative to the data of the 1986 mass tables [24] which include no ISOLTRAP data. Bottom: difference relative to the data of the 1995 mass tables [7] where now the ISOLTRAP mass values are included.

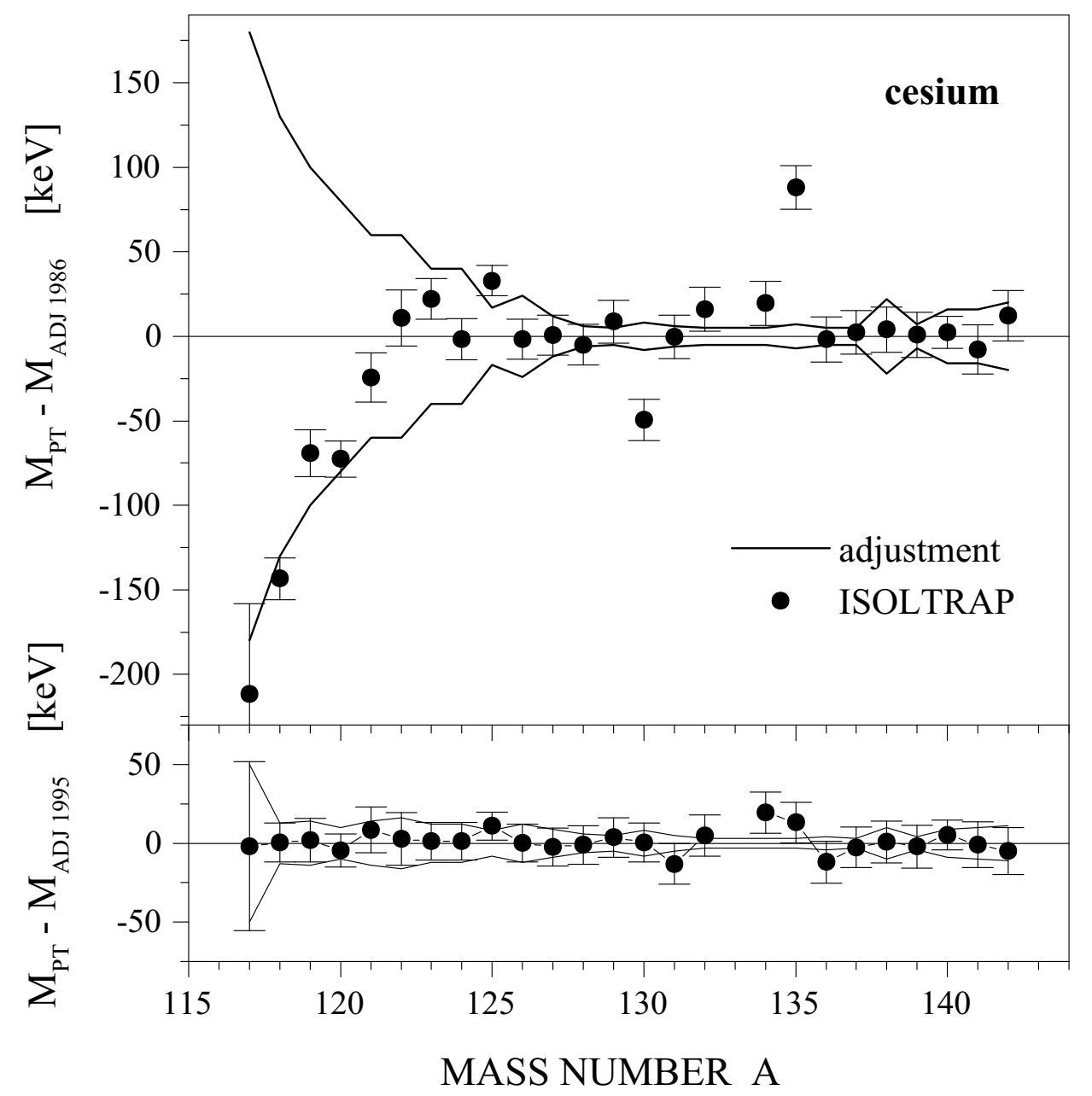




\section{Figure 6}

Same as Fig. 5 but for barium isotopes. Note that the Penning trap data on ${ }^{123,125,127,131} \mathrm{Ba}$ [4] are not included in the 1995 mass update and therefore no adjustment has been made.

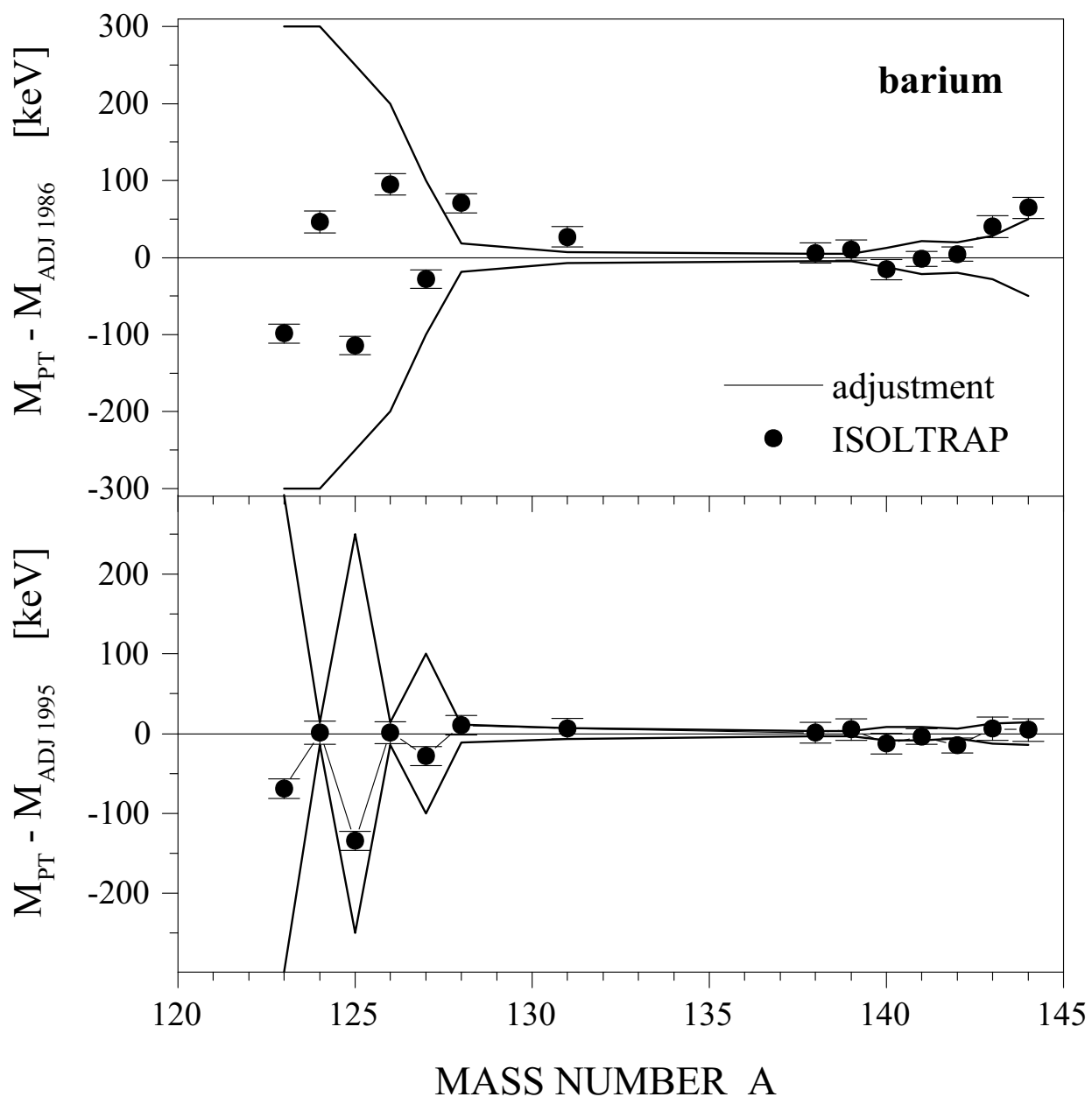




\section{Figure 7}

Comparison of the experimental adjusted masses for Cs (left) and Ba isotopes (right) with various nuclear mass formulae. The difference is plotted versus the mass number. The adjusted masses represent the zero line and their uncertainties are given by the error band. The models are from top to bottom: Jänecke and Masson [36], Duflo and Zuker [39], Möller et al. [40,41], and Pearson et al. [42]. For each model the RMS deviation is given in the figure.

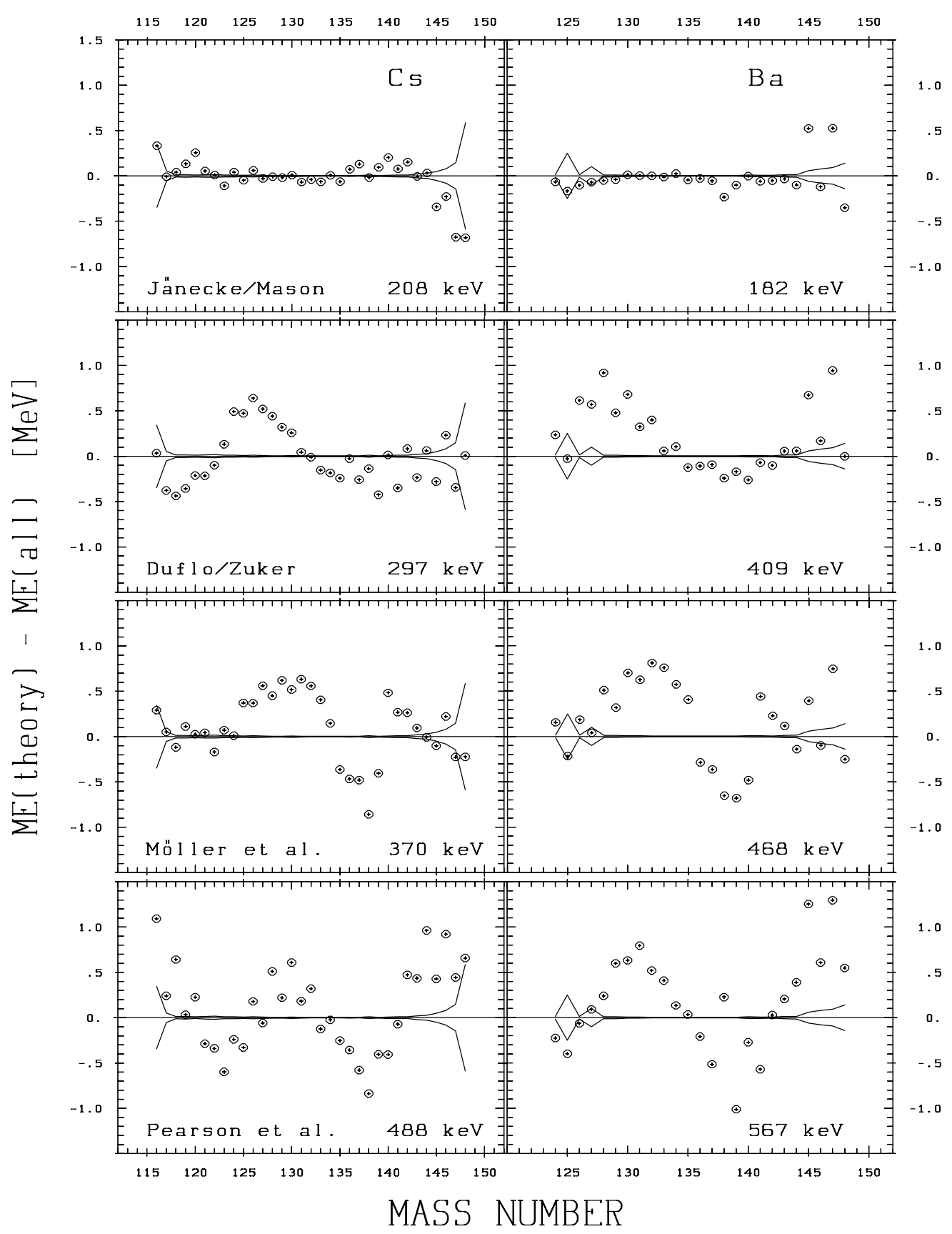




\section{Figure 8}

Comparison of theoretical mass values calculated in the present work with experimental values. Experimental uncertainties are only indicated in the case they exceed the symbol size.

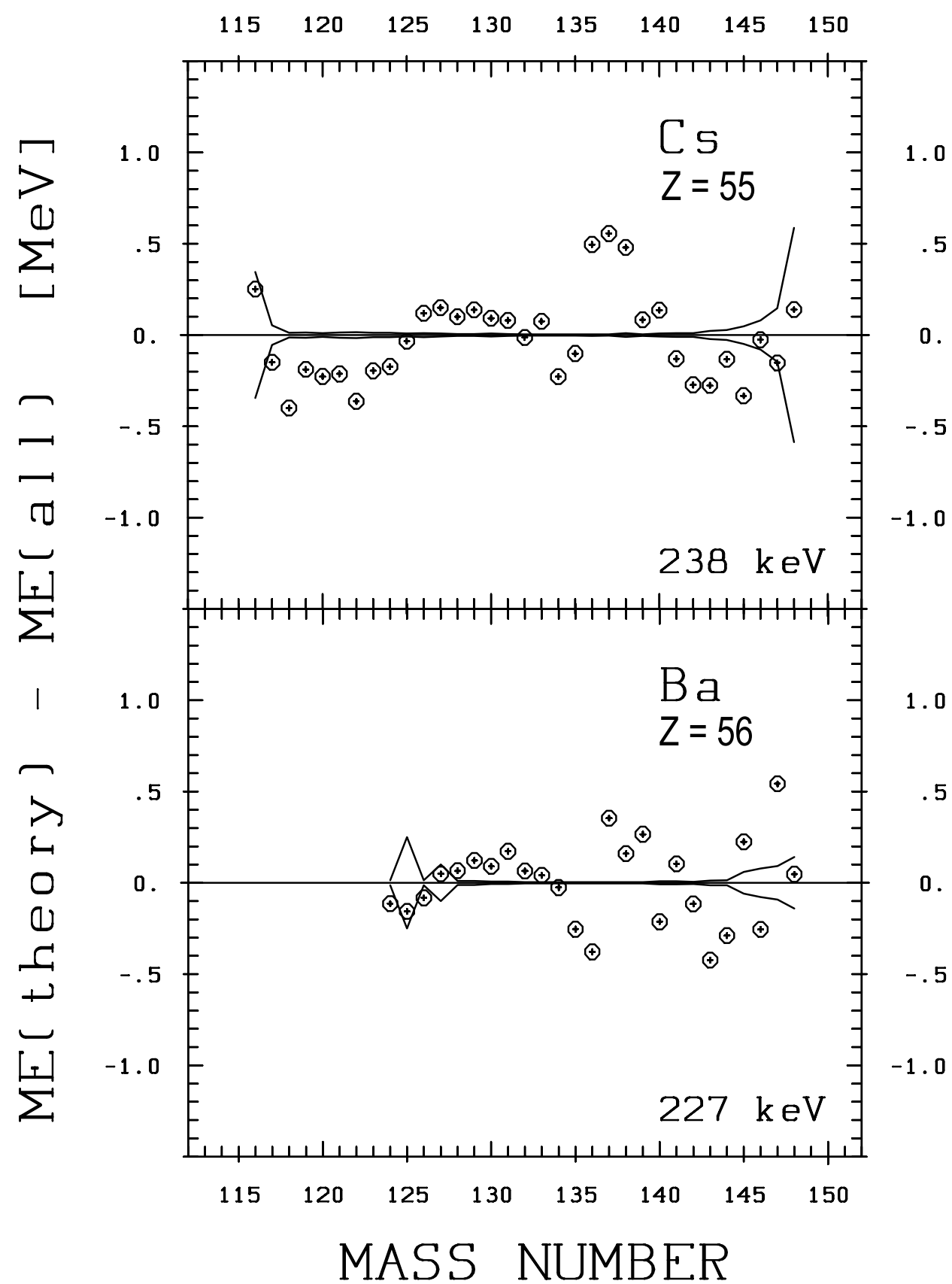




\section{Figure 9}

Comparison of theoretical $\beta_{2}^{\text {th }}$ values (o), as obtained by a macroscopic-microscopic model fitted to experimental mass values, with values $\beta_{2}^{I S}$ extracted from isotope shift measurements $(\triangle)$ and values $\beta_{2}^{Q}$ from hyperfine structure studies $(\diamond)$. The values $\beta_{2}^{\text {th }}$ have been multiplied by a factor of 1.1 (see text). The sign of the $\beta_{2}^{\text {th }}$ and $\beta_{2}^{Q}$ values is shown as part of the symbols.

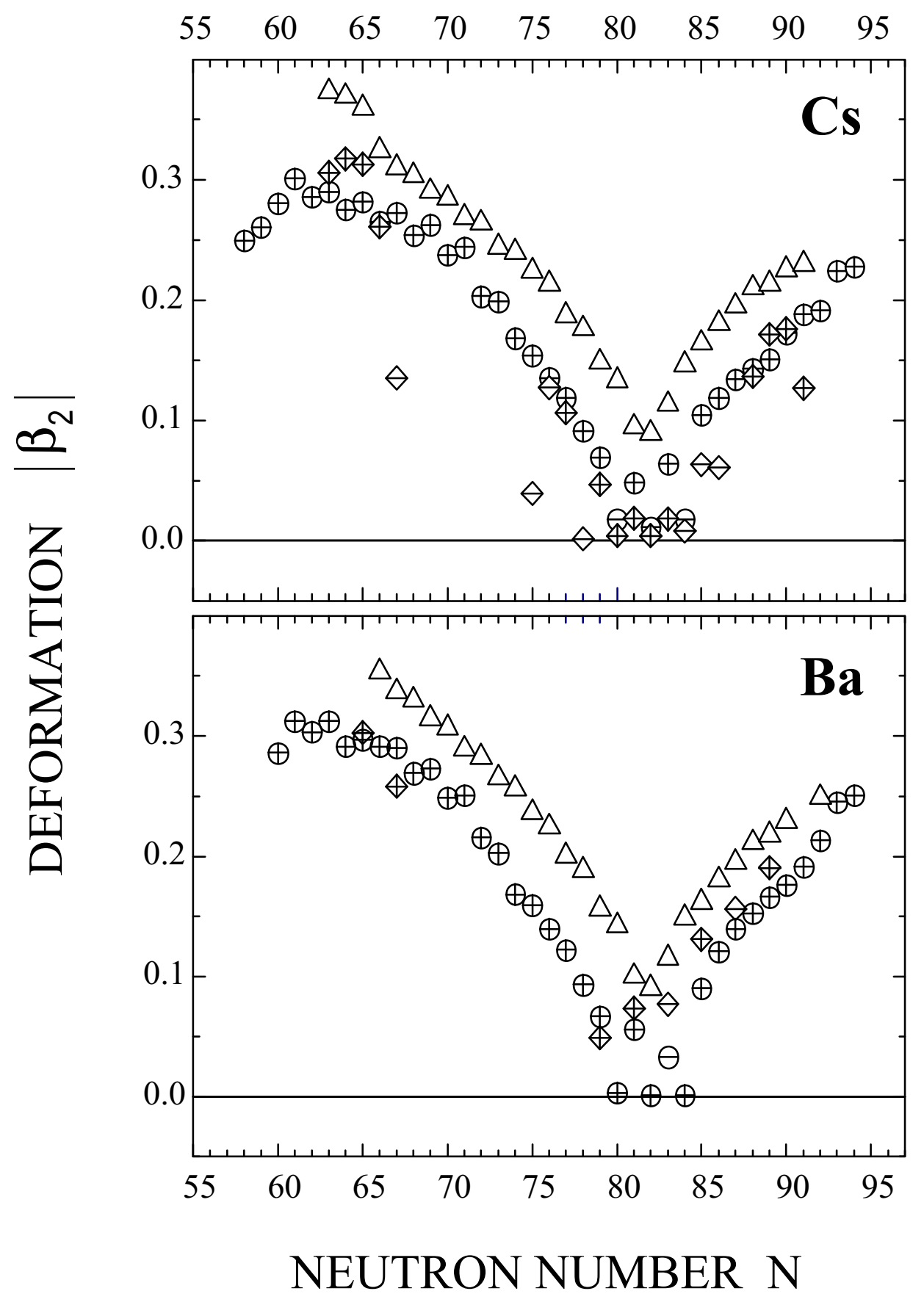




\section{Tables}

Table 1: Target, projectile and surface ionizer used for the production and mass determination of radioactive $\mathrm{Cs}$ and $\mathrm{Ba}$ isotopes at the mass separators ISOLDE-II and PS-Booster ISOLDE in different runs. In all cases, a rhenium foil was used in front of the ISOLTRAP mass spectrometer in order to stop the ISOLDE ion beam and to create a secondary thermal ion beam for capture in the first trap.

\begin{tabular}{cccccl}
\hline & Run & Target & Projectile & Ionizer & Investigated Isotopes \\
\hline \hline ISOLDE-II & $\# 1$ & La & $\mathrm{p}$ & $\mathrm{Ta}$ & ${ }^{118-137} \mathrm{Cs}$ \\
& $\# 2$ & $\mathrm{La}$ & ${ }^{3} \mathrm{He}$ & $\mathrm{W}$ & $123,126,127,129 \mathrm{Cs}{ }^{126,128} \mathrm{Ba}$ \\
& $\# 3$ & $\mathrm{UC}$ & $\mathrm{p}$ & $\mathrm{W}$ & $130,138-140 \mathrm{Cs}{ }^{138-140,142,144} \mathrm{Ba}$ \\
& $\# 4$ & $\mathrm{UC}$ & $\mathrm{p}$ & $\mathrm{W}$ & $137,139,140 \mathrm{Cs}{ }^{124,126,139-144} \mathrm{Ba}$ \\
\hline PSB-ISOLDE & $\# 5$ & $\mathrm{UC}$ & $\mathrm{p}$ & $\mathrm{W}$ & $133,140-142 \mathrm{Cs}{ }^{141,142} \mathrm{Ba}$ \\
\multirow{2}{*}{ new cooler trap } & $\# 6$ & $\mathrm{La}$ & $\mathrm{p}$ & $\mathrm{W}$ & $117,119,120,122,125 \mathrm{Cs}$ \\
new cooler trap & $\# 7$ & Ta & $\mathrm{p}$ & $\mathrm{W}$ & $123,125,127,131 \mathrm{Ba}$ \\
\hline
\end{tabular}


Table 2: Corrections to be applied to the masses measured by ISOLTRAP due to a mixture of nuclei in the ground and isomeric states. The half-lives and spins of ground and isomeric state are indicated. Estimates are marked by \#. The corrections $\Delta E^{*}$ and their uncertainties $\delta\left(\Delta E^{*}\right)$ are taken from the 1993 mass adjustment [25] and the 1995 mass update [7]. In the case of ${ }^{117-120} \mathrm{Cs}$ the assignment of the ground and isomeric states is not certain but based on systematic trends of neighboring nuclides.

\begin{tabular}{|c|c|c|c|c|c|c|}
\hline \multirow[t]{2}{*}{ Isotope } & \multicolumn{2}{|c|}{ g } & \multicolumn{2}{|c|}{$\mathrm{m}$} & \multirow{2}{*}{$\begin{array}{c}\Delta \mathrm{E}^{*}\left(\delta\left(\Delta E^{*}\right)\right) \\
{[\mathrm{keV}]}\end{array}$} & \multirow[t]{2}{*}{ Ref. } \\
\hline & $\mathrm{T}_{1 / 2}$ & $I^{\Pi}$ & $\mathrm{T}_{1 / 2}$ & $I^{\Pi}$ & & \\
\hline${ }^{117} \mathrm{Cs}^{x}$ & $8.4 \mathrm{~s}$ & $9 / 2^{+} \#$ & $6.5 \mathrm{~s}$ & $3 / 2^{+} \#$ & $50(50)$ & {$[7]$} \\
\hline${ }^{118} \mathrm{Cs}^{x}$ & $14 \mathrm{~s}$ & 2 & $17 \mathrm{~s}$ & $7^{-}$ & $5(4)$ & {$[7]$} \\
\hline${ }^{119} \mathrm{Cs}^{x}$ & $43.0 \mathrm{~s}$ & $9 / 2^{+}$ & $30.4 \mathrm{~s}$ & $3 / 2^{+}$ & $16(11)$ & {$[7]$} \\
\hline${ }^{120} \mathrm{Cs}^{x}$ & $57 \mathrm{~s}$ & $2^{(-\#)}$ & $61.2 \mathrm{~s}$ & $7^{-} \#$ & $5(5)$ & {$[7]$} \\
\hline${ }^{121} \mathrm{Cs}^{x}$ & $155 \mathrm{~s}$ & $3 / 2^{+}$ & $122 \mathrm{~s}$ & $9 / 2^{+}$ & $46(8)$ & {$[7]$} \\
\hline${ }^{122} \mathrm{Cs}^{y}$ & $21 \mathrm{~s}$ & $1^{+}$ & $3.7 \mathrm{~min}$ & $8^{-}$ & $61(14)$ & {$[25]$} \\
\hline
\end{tabular}


Table 3: Cyclotron frequency ratios $r=\nu_{r e f} / \nu$ for Cs and Ba ions relative to the cyclotron frequency of ${ }^{133} \mathrm{Cs}^{+}$as obtained with ISOLTRAP at ISOLDE-II. The investigated isotopes are listed in the first column. Superscripts $x$ and $y$ denote isotopes for which a mixture of ground and isomeric state has been investigated. In column 2 the frequency ratios are given. The uncertainties given in column 3 are the statistical errors. The total error given in column 4 includes the estimated maximum systematic error of $1 \cdot 10^{-7}$. Marked by an asterix are isotopes for which a cyclotron frequency ratio was measured in several runs and for which a weighted average of these ratios is given in column 2.

\begin{tabular}{|c|c|c|c|c|}
\hline \multirow{2}{*}{$\begin{array}{l}\text { Isotope } \\
{ }^{118} \mathrm{Cs}^{x}\end{array}$} & \multicolumn{4}{|c|}{ Frequency Ratio $r=\nu_{r e f} / \nu$} \\
\hline & 0.887296204 & $(32)$ & (94) & \\
\hline${ }^{119} \mathrm{Cs}^{x}$ & 0.894789028 & $(36)$ & $(96)$ & \\
\hline${ }^{120} \mathrm{Cs}^{x}$ & 0.902300231 & $(23)$ & (93) & \\
\hline${ }^{121} \mathrm{Cs}^{x}$ & 0.909798622 & $(33)$ & $(97)$ & \\
\hline${ }^{122} \mathrm{Cs}^{y}$ & 0.917314772 & $(16)$ & (93) & \\
\hline${ }^{122} \mathrm{Cs}^{m}$ & 0.917315376 & $(17)$ & (93) & \\
\hline${ }^{123} \mathrm{Cs}$ & 0.924814994 & $(31)$ & $(97)$ & $*$ \\
\hline${ }^{124} \mathrm{Cs}$ & 0.932333566 & $(29)$ & (98) & \\
\hline${ }^{125} \mathrm{Cs}$ & 0.939838867 & $(43)$ & $(103)$ & \\
\hline${ }^{126} \mathrm{Cs}$ & 0.947360862 & $(17)$ & $(96)$ & * \\
\hline${ }^{127} \mathrm{Cs}$ & 0.954869743 & $(14)$ & $(96)$ & * \\
\hline${ }^{128} \mathrm{Cs}$ & 0.962396420 & $(20)$ & $(98)$ & * \\
\hline${ }^{129} \mathrm{Cs}$ & 0.969907961 & $(30)$ & $(102)$ & * \\
\hline${ }^{130} \mathrm{Cs}$ & 0.977436943 & $(14)$ & (99) & * \\
\hline${ }^{131} \mathrm{Cs}$ & 0.984951639 & $(32)$ & $(104)$ & \\
\hline${ }^{132} \mathrm{Cs}$ & 0.992483259 & $(38)$ & $(106)$ & \\
\hline${ }^{134} \mathrm{Cs}$ & 1.007533781 & $(29)$ & $(105)$ & \\
\hline${ }^{135} \mathrm{Cs}$ & 1.015052405 & $(20)$ & (103) & \\
\hline${ }^{136} \mathrm{Cs}$ & 1.022586421 & $(32)$ & (107) & \\
\hline${ }^{137} \mathrm{Cs}$ & 1.030109000 & $(17)$ & $(104)$ & * \\
\hline${ }^{138} \mathrm{Cs}$ & 1.037662754 & $(25)$ & $(107)$ & \\
\hline${ }^{139} \mathrm{Cs}$ & 1.045204564 & $(31)$ & $(109)$ & * \\
\hline${ }^{140} \mathrm{Cs}$ & 1.052758215 & $(27)$ & $(109)$ & * \\
\hline${ }^{124} \mathrm{Ba}$ & 0.932354954 & $(70)$ & $(116)$ & \\
\hline${ }^{126} \mathrm{Ba}$ & 0.947374381 & $(61)$ & $(112)$ & * \\
\hline${ }^{128} \mathrm{Ba}$ & 0.962400730 & $(32)$ & $(101)$ & \\
\hline${ }^{138} \mathrm{Ba}$ & 1.037619346 & $(20)$ & $(106)$ & \\
\hline${ }^{139} \mathrm{Ba}$ & 1.045170598 & $(28)$ & $(108)$ & * \\
\hline${ }^{140} \mathrm{Ba}$ & 1.052707906 & $(17)$ & $(107)$ & * \\
\hline${ }^{141} \mathrm{Ba}$ & 1.060260775 & $(46)$ & $(116)$ & \\
\hline${ }^{142} \mathrm{Ba}$ & 1.067800211 & $(26)$ & $(110)$ & * \\
\hline${ }^{143} \mathrm{Ba}$ & 1.075355959 & $(47)$ & $(117)$ & \\
\hline${ }^{144} \mathrm{Ba}$ & 1.082897612 & $(36)$ & $(114)$ & * \\
\hline
\end{tabular}


Table 4: Cyclotron frequency ratios $r=\nu_{r e f} / \nu$ for Cs, Ba and Ra ions relative to the cyclotron frequency of ${ }^{133} \mathrm{Cs}^{+}$as obtained with ISOLTRAP at the PS-Booster ISOLDE. The investigated isotopes are listed in the first column. Superscripts $x$ and $y$ denote isotopes for which a mixture of ground and isomeric state has been investigated. In column 2 the obtained frequency ratios are given. The uncertainties given in column 3 are the statistical errors. The total error given in column 4 includes the estimated maximum systematic error of $1 \cdot 10^{-7}$.

\begin{tabular}{llrr}
\hline Isotope & Frequency & Ratio $r=\nu_{\text {ref }} / \nu$ \\
\hline \hline${ }^{117} \mathrm{Cs}^{x}$ & 0.879788073 & $(129)$ & $(156)$ \\
${ }^{119} \mathrm{Cs}^{x}$ & 0.894788978 & $(32)$ & $(95)$ \\
${ }^{120} \mathrm{Cs}^{x}$ & 0.902300431 & $(92)$ & $(129)$ \\
${ }^{122} \mathrm{Cs}^{m}$ & 0.917315333 & $(88)$ & $(127)$ \\
${ }^{125} \mathrm{Cs}$ & 0.939838844 & $(42)$ & $(103)$ \\
${ }^{140} \mathrm{Cs}$ & 1.052758368 & $(19)$ & $(107)$ \\
${ }^{141} \mathrm{Cs}$ & 1.060303236 & $(52)$ & $(118)$ \\
${ }^{142} \mathrm{Cs}$ & 1.067859351 & $(57)$ & $(121)$ \\
\hline${ }^{123} \mathrm{Ba}$ & 0.924858523 & $(36)$ & $(99)$ \\
${ }^{125} \mathrm{Ba}$ & 0.939874523 & $(27)$ & $(98)$ \\
${ }^{127} \mathrm{Ba}$ & 0.954897403 & $(20)$ & $(98)$ \\
${ }^{131} \mathrm{Ba}$ & 0.984962860 & $(40)$ & $(106)$ \\
${ }^{141} \mathrm{Ba}$ & 1.060260814 & $(50)$ & $(117)$ \\
${ }^{142} \mathrm{Ba}$ & 1.067800287 & $(48)$ & $(117)$ \\
\hline${ }^{226} \mathrm{Ra}$ & 1.700651167 & $(18)$ & $(171)$ \\
\hline
\end{tabular}


Table 5: Mass excesses as determined by ISOLTRAP. Column 3 lists mass values obtained by combining the frequency ratios given in Table 3 and 4 with the value $\mathrm{ME}=88075.7(3.0) \mathrm{keV}$ [7] for the mass excess of the reference isotope ${ }^{133} \mathrm{Cs}$. Corrected for isomeric contamination (cf Table 2) the data are compared to the mass values listed in the 1986 mass tables (without any Penning trap data) [24] and those of 1995 (with all Penning trap data except for ${ }^{123,125,127,131} \mathrm{Ba}$ ) [7]. The half-lives are taken from Ref. [23]. The last column gives the sum of influences $I F$ of all the Penning trap measurements in the determination of each mass value in the 1995 atomic mass evaluation [7]. Marked with \# are those isotopes for which mass values were not known or where mass estimates from systematic trends were given in the mass tables. 


\begin{tabular}{|c|c|c|c|c|c|c|c|}
\hline \multirow{3}{*}{$\begin{array}{l}\text { Isotope } \\
{ }^{117} \mathrm{Cs}\end{array}$} & \multirow{3}{*}{$\begin{array}{c}T_{1 / 2} \\
\text { (from [23]) }\end{array}$} & \multicolumn{5}{|c|}{ Mass Excess [ keV ] } & \multirow{3}{*}{$\begin{array}{r}\text { IF [\%] } \\
{[7]} \\
100\end{array}$} \\
\hline & & \multirow{2}{*}{$\begin{array}{c}\text { this exp. } \\
-66472(54)\end{array}$} & \multicolumn{2}{|c|}{1986 adj.[24] } & \multicolumn{2}{|c|}{1995 adj.[7] } & \\
\hline & & & -66260 & $(180)$ & -66470 & $(50)$ & \\
\hline${ }^{118} \mathrm{Cs}$ & $14 \mathrm{~s}$ & $-68414(12)$ & -68270 & $(130)$ & -68414 & $(13)$ & 99.8 \\
\hline${ }^{119} \mathrm{Cs}$ & $43.0 \mathrm{~s}$ & $-72309(14)$ & -72240 & $(100)$ & -72311 & $(14)$ & 97 \\
\hline${ }^{120} \mathrm{Cs}$ & $61.2 \mathrm{~s}$ & $-73893(11)$ & -73820 & $(80)$ & -73888 & $(10)$ & 89 \\
\hline${ }^{121} \mathrm{Cs}$ & $155 \mathrm{~s}$ & $-77135(14)$ & -77110 & $(60)$ & -77143 & $(14)$ & 88 \\
\hline${ }^{122} \mathrm{Cs}$ & $21.18 \mathrm{~s}$ & $-78143(18)$ & -78140 & $(60)$ & -78132 & $(16)$ & 83 \\
\hline${ }^{122} \mathrm{Cs}$ & $3.70 \mathrm{~m}$ & $-78010(10)$ & isomer & & -78009 & $(10)$ & 100 \\
\hline${ }^{123} \mathrm{Cs}$ & $5.87 \mathrm{~m}$ & $-81048(12)$ & -81070 & $(40)$ & -81049 & $(12)$ & 97 \\
\hline${ }^{124} \mathrm{Cs}$ & $30.9 \mathrm{~s}$ & $-81742(12)$ & -81740 & $(40)$ & -81743 & $(12)$ & 91 \\
\hline${ }^{125} \mathrm{Cs}$ & $45 \mathrm{~m}$ & -84080 & -84113 & $(17)$ & -84091 & (8) & 66 \\
\hline${ }^{126} \mathrm{Cs}$ & $1.64 \mathrm{~m}$ & $-84349(12)$ & -84347 & $(24)$ & -84349 & $(12)$ & 99 \\
\hline${ }^{127} \mathrm{Cs}$ & $6.25 \mathrm{~h}$ & $-86243(12)$ & -86243 & $(12)$ & -86240 & (9) & 49 \\
\hline${ }^{128} \mathrm{Cs}$ & $3.640 \mathrm{~m}$ & $-85933(12)$ & -85928 & (6) & -85932 & (6) & 20 \\
\hline${ }^{129} \mathrm{Cs}$ & $32.06 \mathrm{~h}$ & $-87498(13)$ & -87506 & $(5)$ & -87501 & (5) & 12 \\
\hline${ }^{130} \mathrm{Cs}$ & $29.21 \mathrm{~m}$ & $-86903(12)$ & -86853 & (8) & -86903 & $(8)$ & 46 \\
\hline${ }^{131} \mathrm{Cs}$ & $9.689 \mathrm{~d}$ & $-88077(13)$ & -88076 & (6) & -88063 & $(5)$ & 15 \\
\hline${ }^{132} \mathrm{Cs}$ & $6.479 \mathrm{~d}$ & $-87155(13)$ & -87171 & $(5)$ & -87160 & (3) & 0 \\
\hline${ }^{133} \mathrm{Cs}$ & stable & reference & -88086 & $(5)$ & $-88075,7$ & (3) & 45 \\
\hline${ }^{134} \mathrm{Cs}$ & $2.0648 \mathrm{y}$ & $-86887(13)$ & -86906 & (5) & $-86905,9$ & (3) & 0 \\
\hline${ }^{135} \mathrm{Cs}$ & $2.3 \mathrm{My}$ & $-87574(13)$ & -87662 & (7) & -87587 & (3) & 0 \\
\hline${ }^{136} \mathrm{Cs}$ & $13.16 \mathrm{~d}$ & $-86356(13)$ & -86354 & (5) & -86344 & (4) & 0 \\
\hline${ }^{137} \mathrm{Cs}$ & $30.07 \mathrm{y}$ & $-86554(13)$ & -86556 & (5) & $-86551,1$ & (3) & 0 \\
\hline${ }^{138} \mathrm{Cs}$ & $33.41 \mathrm{~m}$ & $-82892(13)$ & -82896 & $(22)$ & -82893 & $(10)$ & 48 \\
\hline${ }^{139} \mathrm{Cs}$ & $9.27 \mathrm{~m}$ & $-80709(13)$ & -80710 & $(7)$ & -80707 & $(4)$ & 0 \\
\hline${ }^{140} \mathrm{Cs}$ & $63.7 \mathrm{~s}$ & -77051 & -77053 & $(16)$ & -77056 & (9) & 78 \\
\hline${ }^{141} \mathrm{Cs}$ & $24.94 \mathrm{~s}$ & $-74480(15)$ & -74472 & $(16)$ & -74479 & (10) & 45 \\
\hline${ }^{142} \mathrm{Cs}$ & $1.689 \mathrm{~s}$ & $-70526(15)$ & -70538 & $(20)$ & -70521 & $(11)$ & 47 \\
\hline${ }^{123} \mathrm{Ba}$ & $2.7 \mathrm{~m}$ & $-75659(12)$ & -75560 & 300) \# & $-75590(3$ & 00) \# & 100 \\
\hline${ }^{124} \mathrm{Ba}$ & $11.9 \mathrm{~m}$ & $-79094(14)$ & -79140 & 300) \# & -79095 & $(14)$ & 100 \\
\hline${ }^{125} \mathrm{Ba}$ & $3.5 \mathrm{~m}$ & $-79665(12)$ & -79550 & $(250)$ & -79530 & $(250)$ & 100 \\
\hline${ }^{126} \mathrm{Ba}$ & $100 \mathrm{~m}$ & $-82675(14)$ & -82770 & 200) \# & -82676 & (14) & 100 \\
\hline${ }^{127} \mathrm{Ba}$ & $12.7 \mathrm{~m}$ & $-82818(12)$ & -82790 & $(100)$ & -82790 & $(100)$ & 100 \\
\hline${ }^{128} \mathrm{Ba}$ & $2.43 \mathrm{~d}$ & $-85400(13)$ & -85470 & (18) & -85410 & (11) & 81 \\
\hline${ }^{131} \mathrm{Ba}$ & $11.50 \mathrm{~d}$ & $-86687(13)$ & -86714 & (7) & -86693 & (7) & 23 \\
\hline${ }^{138} \mathrm{Ba}$ & stable & $-88266(13)$ & -88272 & (5) & $-88267,2$ & (3) & 0 \\
\hline${ }^{139} \mathrm{Ba}$ & $83.06 \mathrm{~m}$ & $-84914(13)$ & -84924 & $(5)$ & $-84919,3$ & (3) & 0 \\
\hline${ }^{140} \mathrm{Ba}$ & $12.752 \mathrm{~d}$ & $-83289(13)$ & -83273 & $(12)$ & -83276 & (8) & 37 \\
\hline${ }^{141} \mathrm{Ba}$ & $18.27 \mathrm{~m}$ & $-79734(10)$ & -79732 & $(21)$ & -79730 & (8) & 65 \\
\hline${ }^{142} \mathrm{Ba}$ & $10.6 \mathrm{~m}$ & $-77843(10)$ & -77847 & $(20)$ & -77828 & (6) & 37 \\
\hline${ }^{143} \mathrm{Ba}$ & $14.33 \mathrm{~s}$ & $-73939(14)$ & -73979 & $(28)$ & -73945 & (13) & 76 \\
\hline${ }^{144} \mathrm{Ba}$ & $11.5 \mathrm{~s}$ & $-71776(14)$ & -71840 & $(50)$ & -71780 & (14) & 93 \\
\hline${ }^{226} \mathrm{Ra}$ & $1.600 \mathrm{ky}$ & $23678 \quad(22)$ & -23662.6 & $(2.7)$ & 23662.3 & $(2.5)$ & 3 \\
\hline
\end{tabular}


Table 6: Adjusted parameters of the microscopic-macroscopic model used in this work. A comparison is made with the macroscopic parameters from refs. [46], [51] and with the pairing parameters for the Lipkin-Nogami-approach from [40].

\begin{tabular}{lccccc}
\hline & $\kappa_{v}$ & $c_{a}[\mathrm{MeV}]$ & $c_{0}[\mathrm{MeV}]$ & $r[\mathrm{MeV}]$ & $t$ \\
\hline \hline This work, Cs: & 1.937 & 0.25 & 4.1 & 1.45 & 0 \\
This work, Ba: & 1.955 & 0.31 & 4.1 & 2.2 & 9 \\
\hline Ref. [40,46]: & 1.927 & 0.212 & 4.4 & 3.3 & 0 \\
Ref. [51]: & 1.962 & 0.33 & 4.4 & - & - \\
\hline
\end{tabular}


Table 7: Results of the conversion from cyclotron frequency ratios given in Table 3 into linear relations for mass adjustment. The equations representing the ISOLTRAP data are listed in the first column, where the symbol for the nuclides refer to their mass excesses. The second column gives the experimental values for these relations in $\mu \mathrm{u}$. Their adjusted values in the least squares fit in the 1995 Atomic Mass Evaluation [7] are given in the third column. In column 4 the relative deviations $v / s$ between measured and adjusted values are given as their differences $v$ divided by the uncertainty $s$ of the experimental value. The last column gives the significance $S$ [35] of the present data in the new adjustment. The "Partial Consistency Factor" [61] of this set of data is 0.95 . 


\begin{tabular}{|c|c|c|c|c|c|}
\hline \multicolumn{6}{|c|}{ Cesium } \\
\hline linear relation & exp. value $[\mu \mathrm{u}]$ & adj. valu & ue $[\mu \mathrm{u}]$ & $v / s$ & $S$ \\
\hline${ }^{118} \mathrm{Cs}^{x}-0.887 \cdot{ }^{133} \mathrm{Cs}$ & $10429(13)$ & 10429 & (13) & 0.0 & $100 \%$ \\
\hline${ }^{119} \mathrm{Cs}^{x}-0.895 \cdot{ }^{133} \mathrm{Cs}$ & $7018(13)$ & 7013 & (9) & -0.4 & $49 \%$ \\
\hline${ }^{120} \mathrm{Cs}^{x}-0.902 \cdot{ }^{133} \mathrm{Cs}$ & $5956(12)$ & 5970 & (9) & 1.2 & $60 \%$ \\
\hline${ }^{121} \mathrm{Cs}^{x}-0.910 \cdot{ }^{133} \mathrm{Cs}$ & $3285(13)$ & 3276 & (12) & -0.7 & $88 \%$ \\
\hline${ }^{122} \mathrm{Cs}^{m}-0.917 \cdot{ }^{133} \mathrm{Cs}$ & $2961(12)$ & 2959 & $(10)$ & -0.2 & $67 \%$ \\
\hline${ }^{122} \mathrm{Cs}^{y}-0.917 \cdot{ }^{133} \mathrm{Cs}$ & $2880(12)$ & 2885 & $(12)$ & 0.4 & $92 \%$ \\
\hline${ }^{123} \mathrm{Cs} \quad-0.925 \cdot{ }^{133} \mathrm{Cs}$ & $453(13)$ & 452 & $(13)$ & -0.1 & $97 \%$ \\
\hline${ }^{124} \mathrm{Cs} \quad-0.932 \cdot{ }^{133} \mathrm{Cs}$ & $370(13)$ & 369 & $(12)$ & -0.1 & $91 \%$ \\
\hline${ }^{125} \mathrm{Cs} \quad-0.940 \cdot{ }^{133} \mathrm{Cs}$ & $-1382(14)$ & -1395 & (8) & -0.9 & $34 \%$ \\
\hline${ }^{126} \mathrm{Cs} \quad-0.947 \cdot{ }^{133} \mathrm{Cs}$ & $-1011(13)$ & -1010 & $(13)$ & 0.1 & $99 \%$ \\
\hline${ }^{127} \mathrm{Cs} \quad-0.955 \cdot{ }^{133} \mathrm{Cs}$ & $-2287(13)$ & -2284 & (9) & 0.2 & $52 \%$ \\
\hline${ }^{128} \mathrm{Cs} \quad-0.962 \cdot{ }^{133} \mathrm{Cs}$ & $-1293(13)$ & -1292 & $(6)$ & 0.1 & $24 \%$ \\
\hline${ }^{129} \mathrm{Cs} \quad-0.970 \cdot{ }^{133} \mathrm{Cs}$ & $-2216(14)$ & -2220 & $(6)$ & -0.3 & $16 \%$ \\
\hline${ }^{130} \mathrm{Cs} \quad-0.977 \cdot{ }^{133} \mathrm{Cs}$ & $-916(13)$ & -915 & (9) & 0.0 & $49 \%$ \\
\hline${ }^{131} \mathrm{Cs} \quad-0.985 \cdot{ }^{133} \mathrm{Cs}$ & $-1419(14)$ & -1404 & $(6)$ & 1.0 & $19 \%$ \\
\hline${ }^{132} \mathrm{Cs} \quad-0.992 \cdot{ }^{133} \mathrm{Cs}$ & $232(14)$ & 226.5 & $(2.1)$ & -0.4 & $0 \%$ \\
\hline${ }^{134} \mathrm{Cs} \quad-1.008 \cdot{ }^{133} \mathrm{Cs}$ & $2033(14)$ & 2023.01 & $(0.03)$ & -0.7 & $0 \%$ \\
\hline${ }^{135} \mathrm{Cs}-1.015 \cdot{ }^{133} \mathrm{Cs}$ & $1957(14)$ & 1943.4 & $(1.1)$ & -1.0 & $0 \%$ \\
\hline${ }^{136} \mathrm{Cs} \quad-1.023 \cdot{ }^{133} \mathrm{Cs}$ & $4021(14)$ & 4033.6 & $(2.1)$ & 0.9 & $0 \%$ \\
\hline${ }^{137} \mathrm{Cs} \quad-1.030 \cdot{ }^{133} \mathrm{Cs}$ & $4470(14)$ & 4473.2 & $(0.7)$ & 0.2 & $0 \%$ \\
\hline${ }^{138} \mathrm{Cs} \quad-1.038 \cdot{ }^{133} \mathrm{Cs}$ & $9157(14)$ & 9157 & $(10)$ & 0.0 & $49 \%$ \\
\hline${ }^{139} \mathrm{Cs} \quad-1.045 \cdot{ }^{133} \mathrm{Cs}$ & $12163(14)$ & 12166 & $(3)$ & 0.2 & $0 \%$ \\
\hline${ }^{140} \mathrm{Cs} \quad-1.053 \cdot{ }^{133} \mathrm{Cs}$ & $16836(14)$ & 16842 & $(9)$ & 0.4 & $39 \%$ \\
\hline
\end{tabular}


Table 7 continued:

\begin{tabular}{cccccc}
\hline \multicolumn{5}{c}{ Barium } \\
\hline linear relation & exp. value $[\mu \mathrm{u}]$ & adj. value $[\mu \mathrm{u}]$ & $v / s$ & $S$ \\
\hline \hline${ }^{124} \mathrm{Ba}-0.932 \cdot{ }^{133} \mathrm{Cs}$ & $3212(15)$ & & & & $100 \%$ \\
${ }^{126} \mathrm{Ba}-0.947 \cdot{ }^{133} \mathrm{Cs}$ & $786(15)$ & & & & $100 \%$ \\
${ }^{128} \mathrm{Ba}-0.962 \cdot{ }^{133} \mathrm{Cs}$ & $-720(13)$ & -731 & $(12)$ & -0.8 & $81 \%$ \\
${ }^{138} \mathrm{Ba}-1.038 \cdot{ }^{133} \mathrm{Cs}$ & $3388(14)$ & 3388.4 & $(0.7)$ & 0.0 & $0 \%$ \\
${ }^{139} \mathrm{Ba}-1.045 \cdot{ }^{133} \mathrm{Cs}$ & $7649(14)$ & 7643.4 & $(0.7)$ & -0.4 & $0 \%$ \\
${ }^{140} \mathrm{Ba}-1.053 \cdot{ }^{133} \mathrm{Cs}$ & $10150(14)$ & 10164 & $(9)$ & 1.0 & $37 \%$ \\
${ }^{141} \mathrm{Ba}-1.060 \cdot{ }^{133} \mathrm{Cs}$ & $14625(15)$ & 14633 & $(8)$ & 0.5 & $31 \%$ \\
${ }^{142} \mathrm{Ba}-1.068 \cdot{ }^{133} \mathrm{Cs}$ & $17410(15)$ & 17431 & $(7)$ & 1.4 & $19 \%$ \\
${ }^{143} \mathrm{Ba}-1.075 \cdot{ }^{133} \mathrm{Cs}$ & $22268(16)$ & 22262 & $(14)$ & -0.4 & $76 \%$ \\
${ }^{144} \mathrm{Ba}-1.083 \cdot{ }^{133} \mathrm{Cs}$ & $25347(15)$ & 25342 & $(14)$ & -0.4 & $93 \%$ \\
\hline
\end{tabular}


Table 8: Results of the conversion from cyclotron frequency ratios given in Table 4 into linear relations. These data refer to more recent independent measurements performed at the PSB-ISOLDE. For description of the different columns see Table 7. The "Partial Consistency Factor" [61] of this set of data is 0.92 .

\begin{tabular}{|c|c|c|c|c|}
\hline \multicolumn{5}{|c|}{ Cesium } \\
\hline linear relation & exp. value $[\mu \mathrm{u}]$ & adj. value $[\mu \mathrm{u}]$ & $v / s$ & $S$ \\
\hline${ }^{117} \mathrm{Cs}^{x}-0.880 \cdot{ }^{133} \mathrm{Cs}$ & $11900(21)$ & & & $100 \%$ \\
\hline${ }^{119} \mathrm{Cs}^{x}-0.895 \cdot{ }^{133} \mathrm{Cs}$ & $7012(13)$ & 7013 & 0.1 & $49 \%$ \\
\hline${ }^{120} \mathrm{Cs}^{x}-0.902 \cdot{ }^{133} \mathrm{Cs}$ & $5983(17)$ & $5970 \quad(9)$ & -0.7 & $30 \%$ \\
\hline${ }^{122} \mathrm{Cs}^{m}-0.917 \cdot{ }^{133} \mathrm{Cs}$ & $2955(17)$ & $2959 \quad(10)$ & 0.2 & $33 \%$ \\
\hline${ }^{125} \mathrm{Cs} \quad-0.940 \cdot{ }^{133} \mathrm{Cs}$ & $-1386(14)$ & -1395 & -0.7 & $34 \%$ \\
\hline${ }^{133} \mathrm{Cs} \quad-0.588 \cdot{ }^{226} \mathrm{Ra}$ & $-109500(13)$ & $-109490 \quad(3)$ & 0.8 & $7 \%$ \\
\hline${ }^{140} \mathrm{Cs}-1.053 \cdot{ }^{133} \mathrm{Cs}$ & $16857(14)$ & 16842 & -1.1 & $39 \%$ \\
\hline${ }^{141} \mathrm{Cs} \quad-1.060 \cdot{ }^{133} \mathrm{Cs}$ & $20269(16)$ & $20270(11)$ & 0.1 & $46 \%$ \\
\hline${ }^{142} \mathrm{Cs}-1.068 \cdot{ }^{133} \mathrm{Cs}$ & $25270(16)$ & $25275(11)$ & 0.3 & $47 \%$ \\
\hline \multicolumn{5}{|c|}{ Barium } \\
\hline linear relation & exp. value $[\mu \mathrm{u}]$ & adj. value $[\mu \mathrm{u}]$ & $v / s$ & $S$ \\
\hline${ }^{141} \mathrm{Ba}-1.060 \cdot{ }^{133} \mathrm{Cs}$ & $14631(16)$ & $14633(8)$ & 0.1 & $31 \%$ \\
\hline${ }^{142} \mathrm{Ba}-1.068 \cdot{ }^{133} \mathrm{Cs}$ & $17420(16)$ & $17431(7)$ & 0.8 & $17 \%$ \\
\hline
\end{tabular}

